\title{
Autonomy in Surgical Robotics
}

\author{
Aleks Attanasio \\ School of Electronic and \\ Electrical Engineering, \\ University of Leeds, UK \\ Email: elaat@leeds.ac.uk \\ Elena De Momi \\ Dipartimento di Elettronica, \\ Informazione e Bioingegneria, \\ Politecnico di Milano, Italy, \\ Email: elena.demomi@polimi.it
}

\author{
Bruno Scaglioni \\ School of Electronic and \\ Electrical Engineering, \\ University of Leeds, UK \\ Email: b.scaglioni@leeds.ac.uk
}

\author{
Paolo Fiorini \\ Department of Computer Science, \\ Università di Verona, Italy, \\ Email: paolo.fiorini@univr.it
}

\author{
Pietro Valdastri \\ School of Electronic and \\ Electrical Engineering, \\ University of Leeds, UK \\ Email: p.valdastri@leeds.ac.uk
}

\begin{abstract}
Stemming from the dichotomy between "automatic" and "autonomous" behaviour of a surgical robot, this review maps the levels of autonomy and lists the main enabling technologies that are driving the research in this field. The paper is organized in five main sections, according to increasing levels of autonomy. At Level-0, where the bulk of commercial platforms is, the robot is not allowed any autonomy. At Level-1, the robot can provide cognitive and physical assistance to the surgeon, while at Level-2, it can autonomously perform a surgical task. Level-3 comes with conditional autonomy, providing the robot with the ability to plan a task and update planning during execution. Finally, the robot at Level-4 can plan and execute a sequence of surgical tasks autonomously.
\end{abstract}

\section{Introduction}

Surgical robotics is one of the most successful areas of robotics, with great acceptance, impressive economic return and very active research and development communities. This area lays at the intersection of three very different communities, each with its own peculiar perspective: surgeons look at robots as advanced tools that help them augment their capabilities, engineers like to explore these very challenging and rewarding applications, and entrepreneurs see this area as a great business opportunity. These different points of view may help to explain some of the apparently contradicting developments in the field, such as the large divide between the number of excellent prototypes developed in research laboratories and the relative small number of platforms that made it to the market. The long certification process and the associated high costs do not discourage researchers from implementing new ideas and testing new prototypes in spite of the minimal chance that their devices will ever be used in clinical practice. Where these three communities must converge is on establishing the basic scientific and technological principles guiding current and future developments. This is the case, for instance, of autonomous surgical robots, a challenging field that is now spurring much excitement in research laboratories and has a great future potential, once its regulatory and legal issues are properly addressed. A first proposal to structure these research efforts was the Editorial "Medical robotics-Regulatory, ethical, and legal considerations for increasing levels of autonomy", appeared on Science Robotics in 2017 [1] that classified the autonomy achievable by a surgical robot into six levels: no autonomy, robot assistance, task autonomy, conditional autonomy, high autonomy, and full autonomy. This classification is inspired by the "Automated Driving" level definition in the field of automotive [2], and adapts these concepts to robotic surgery. However, the transition from the road to the operating room is characterized by additional complexity. So, while 
autonomous driving has reached Level-3 of autonomy, approaching four, robotic surgery is still solidly resting at Level-0 for what concerns commercially available platforms. This could be explained by the differences in investments and number of players in the two fields, with surgical robotics dominated by few disproportionately large companies that have no rush in disrupting the status quo.

A second important clarification that needs to be made is the difference between "automatic" and "autonomous" behaviors. Automatic behaviors are completely predictable, as they follow well established theories, either deterministic or probabilistic. Although there are variations of behaviors for an automatic system, these are due to small adaptations of the controller parameters to external conditions. If variations are too large, an automatic system cannot adapt and consequently fails. An autonomous system instead, is able to make large adaptations to a change in the external conditions by planning its tasks. The planning function requires a wider domain knowledge and the use of cognitive tools, e.g. ontologies or logical rules that do not exist within an automatic system.

The quest for some level of autonomy in robotics has received a boost by the current COVID-19 pandemic, which has made many familiar places, especially hospitals and schools, hostile environments where robots, rather than humans, should operate. During the pandemic's peak, all but the emergency surgical interventions have been suspended due to the increased risks of virus transmission for patients and medical staff. In this context, surgical robots that can be teleoperated from a safe distance, or mobile robots capable of disinfecting a room or carrying out simple autonomous tasks in the hospital would have been extremely beneficial. All these activities need some form of autonomy, either to compensate communication delays and interruptions in tele-surgery, or to plan an appropriate cleaning path, or to interact intelligently with the human operators. These desired capabilities nicely map to the levels of autonomy defined in [1] and could inject new resources and ideas into autonomous robotics research. In robotic surgery however, the levels of autonomy have not been clearly mapped to specific surgical functions and, so far, there is an ample debate on which level of autonomy is appropriate for a given surgical task.

This paper is motivated by the desire to help both robotics and medical researchers to identify the most appropriate autonomous functions for their application, and discuss the technologies that have already been developed towards the implementation of the desired autonomy level. The first goal of this paper is to draw a clear map of levels of autonomy and surgical actions, since the terms used so far can be ambiguous and perhaps too vague. Second, we propose a comprehensive review of the enabling technologies for autonomous surgical robotics, since most of them are available already, but their sensible integration is often still missing. Finally, we aim at providing robotics researchers with a guide to the different aspects of autonomous surgery, by presenting approaches that, in our opinion, stand in the field as more successful or promising.

The paper is organized following the structure of the levels of autonomy presented in [1], limiting our investigation to the first five levels, because no relevant examples exist so far for a fully autonomous surgical robot. For each level of autonomy, we describe the enabling technologies and the specific surgical actions that can be performed at that level. Section 2 provides a brief overview of commercially available platforms, all of them possessing no autonomy, referring the interested reader to some of the many reviews already available. Section 3 discusses the first level of autonomy in which a robot is able to provide either cognitive or physical assistance to the surgeon. In our opinion, this is the most crucial step in technology development, as it aims at achieving surgical situation awareness and providing specific assistance, serving as the foundation for all next levels. The second level refers to task autonomy, i.e. when a surgical procedure can be represented by a discrete sequence of tasks, a surgical robot operating at this level is able to carry out autonomously one of the tasks, but it has neither an understanding of the full surgical plan, nor the capability to alter task planning during execution. Section 5 addresses the third level of autonomy, i.e. "conditional autonomy", where the surgical robot is able to make "local" adjustments to a pre-defined surgical plan and determine which task to execute next based on its own pre-operative and intra-operative understanding. The last level of autonomy addressed by the paper is Level-4, where the surgical robot is able to interpret pre-operative and intra-operative information, devise a surgical plan and execute it autonomously, re-planning if necessary. The summary of the available enabling technologies and practical applications discussed in this paper is detailed in Figure 1 along with their readiness for the implementation in a real surgical scenario.

The inclusion of levels of autonomy in a surgical robot, or in any robot for that matter, raises a number of important questions regarding the Ethical, Legal, Social and Economic aspects of autonomy, usually referred to as "ELSE" aspects. A detailed discussion of them is beyond the scope of this paper, but we cannot avoid raising a few flags on the importance of making the robotics research community aware of the profound ELSE impacts of autonomous technologies, as discussed in section 7. Fortunately we believe that, in the medical and surgical arenas, autonomous robots will have mostly a beneficial impact, by reducing workload of medical personnel and costs for the hospitals, while potentially increasing patient safety and quality of healthcare delivery. However, we need to carefully communicate to the general public and make every effort to avoid the impression that hospitals will become an "assembly line" in the healthcare systems of the future.

\section{Level 0 - No autonomy}

[Definition of Level 0] Systems at level-0 are not autonomous, the motion of the robot is controlled exclusively by the surgeon, no supports or constraints are provided. 


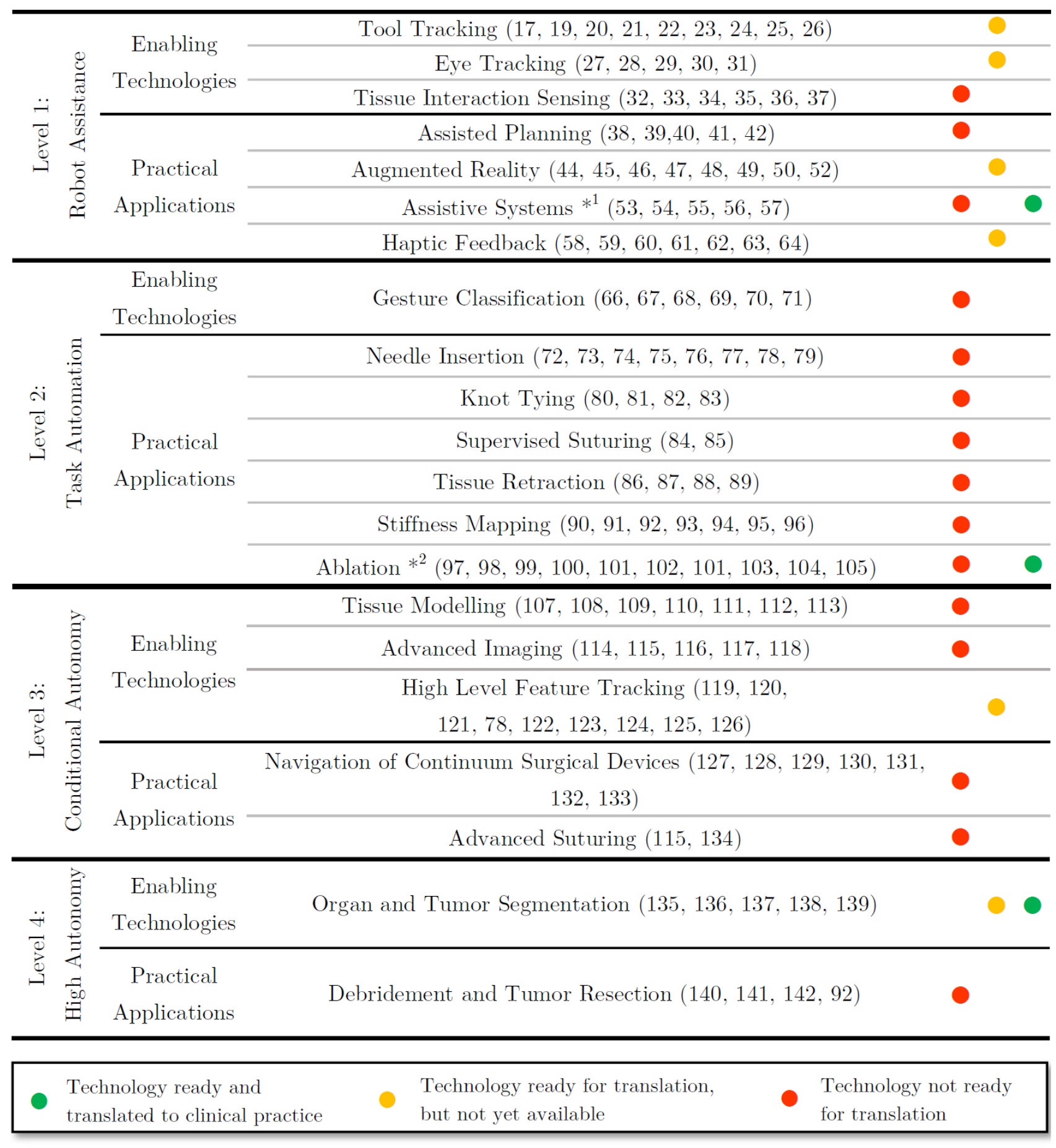

Fig. 1. Readiness level of enabling technologies and research areas for the different levels of autonomy. $*^{1}$ in orthopaedics the problem of assistive systems is solved for specific applications (see the Mako, Stryker for joint replacement), the problem is not solved yet for soft tissue surgery. $*^{2}$ Ablation for specific application such as the treatment of benign prostatic hyperplasia is a commercially solved problem (see AquaBeam, Procept Biorobotics), however, ablation in endoscopic surgery is still matter of research. $*^{3}$ In neurosurgery the segmentation of tumors from MRI images is already implemented in the Brainlab technology. The challenge remains open for thoracic and abdominal surgery.

A vast amount of literature is available on systems at Level-0, often aimed at describing the commercial scenario and the platforms in a translational stage. The literature suggests a great commercial interest in the field of surgical robotics. The expiration of several patents owned by Intuitive Surgical has attracted the interests of venture capitals, consequently triggering the inception of many new robotic platforms. In year 2000, the DaVinci system from Intuitive Surgical introduced the paradigm of transparent teleoperation, where movements performed by the surgeon on the control interface are exactly 


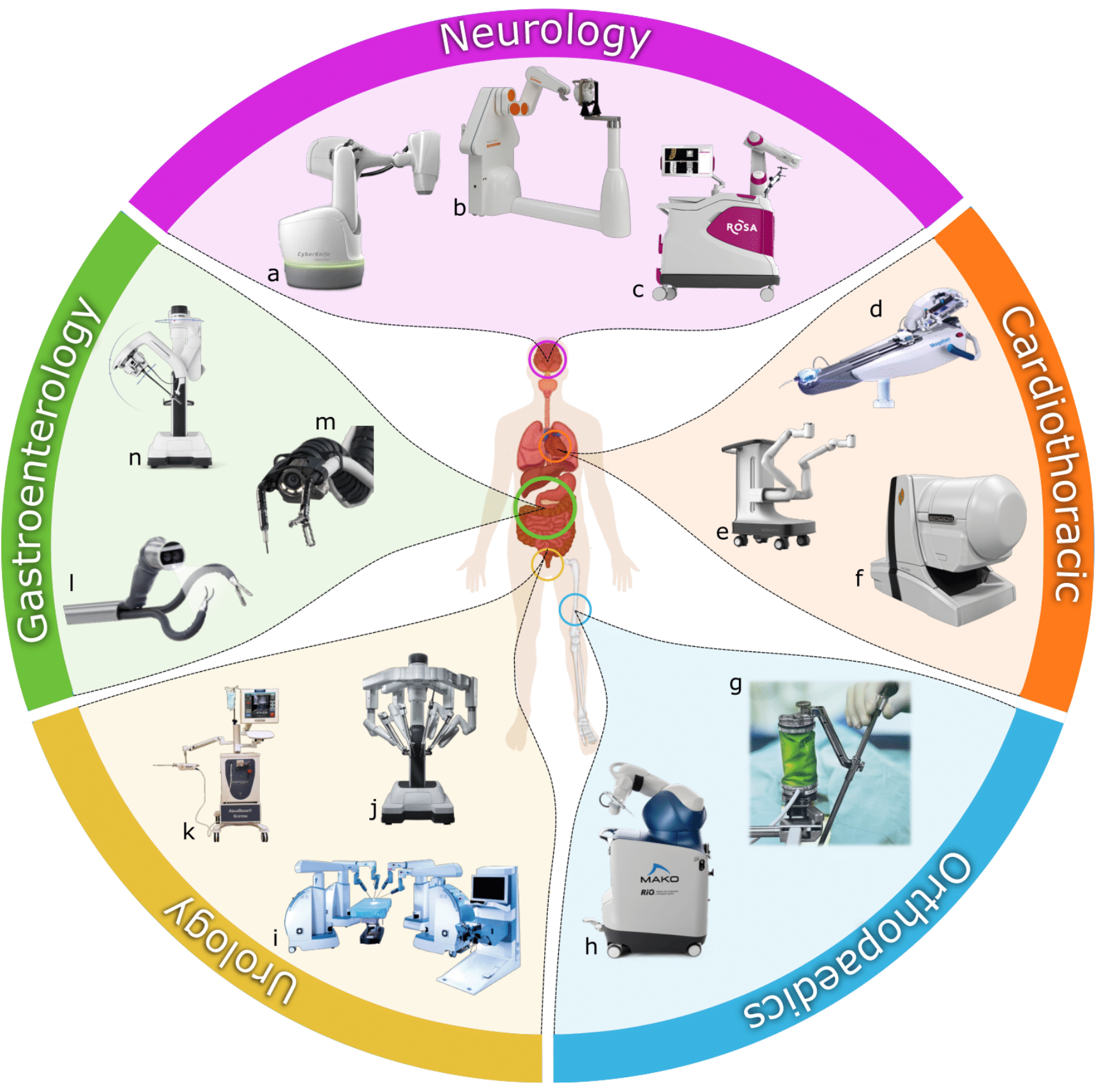

Fig. 2. Commercially available systems organised by clinical application: (a) CyberKnife, M6 (b) NeuroMate, Renishaw (c) ROSA ONE, Zimmer Biomet Robotics (d) Magellan, Hansen Medical Inc. (e) Monarch, Auris Health (f) Niobe, Stereotaxis (g) Renaissance, Mazor Robotics (h) Mako, Stryker (i) Senhance, Transenterix (j) Da Vinci Xi, Intuitive Surgical (k) AquaBeam, Procept BioRobotics (I) SPORT, Titan Medical (m) Flex Robotic System, Medrobotics (n) Da Vinci SP, Intuitive Surgical

replicated by the surgical instruments on the patient side. This is the main characteristic of the Level- 0 devices and the key feature that led to regulatory approval of surgical robotic platforms so far. The absence of a decision-making process by the machine in the transparent teleoperation paradigm leaves complete control to the surgeon. This feature has allowed Intuitive Surgical to claim that, in the absence of technical failures, the responsibility is totally held by the surgeon. This approach resulted in just two lawsuits reaching trial out of more than 3000 filed against the company up to 2016. Recently, several lawsuits have accused Intuitive of not properly training medical staff members, but all the actions have been resolved with settlements. In light of the vast amount of literature available and the specific focus of this paper on autonomy, we will only provide a brief overview of Level-0 platforms by citing existing review papers in the field. The interested reader can refer to [3] for an exhaustive list of surgical robotic platforms approved by the American Food and Drug Administration (FDA) as of 2018. In [4], a comprehensive description of the commercial systems intended for research purposes as of 2015 is provided. The platforms are classified in commercially available / developed for commercial use (but not on the market) and 
advanced research prototypes.

Several reviews are dedicated to specific sub-fields; in 2010 [5] provided a detailed description of micro-robots for surgical applications. The paper highlights how the target surgery (ranging from drug delivery to vessels repair) influences the design. The miniaturization trend is described in [6], where the research platforms are mapped on a decreasing dimensional scale. The paper predicts a considerable spread of intracorporeal devices, aimed at tackling pathologies at a cellular level. In a review published in 2013 [7], the authors map platforms in relation to their access route into the patient's body (intralumenal, extralumenal, translumenal and hybrid). For each category, strengths and weaknesses are discussed, along with the open challenges in each particular field.

Another popular classification method is toward clinical applications; in a 2018 review [8], robotic systems for otologic surgery are described, highlighting the need for a robot able to perform cholesteatoma surgery and indicating miniaturization as the main technical issue yet to be solved. In the field of neurosurgery, [9] provides a comprehensive review of the available systems as of 2016, while [10] reviews the available robotic systems for stereo-tactic approaches. From a different perspective, [11] reports an interesting cross section of the evolution of surgical robotic systems, starting from the first commercially available devices: the voice-controlled endoscopic holders AESOP and ZEUS, both discontinued. In the field of colorectal surgery, [12] reviews flexible devices for ednolumenal and translumenal interventions, making a distinction between mechanical and robotic systems, and concluding that mechanical design of both would require massive upgrades to address the clinical needs of surgical endoscopy. Interestingly, no technical review is available in the field of urology, as the Intuitive's daVinci robot is dominating the field. As new competitors (e.g. Cambridge Medical Robotics, Transenterix) enter the market, reviews comparing robotic platforms for abdominal surgery in general, and urology in particular, may be expected.

The literature reviewed in this section suggests a clear trend: while the commercial scenario is flourishing in many directions, the research at Level-0 is focused on development of platforms for unmet clinical needs, such as microsurgery [13], endoscopic intervention [14] and MRI-compatible surgery [15]. On the other hand, for clinical applications where robotics is already well established (e.g. abdominal surgery), research is progressing towards higher levels of autonomy, as discussed in the following sections of the paper.

\section{Level 1 - Robot Assistance}

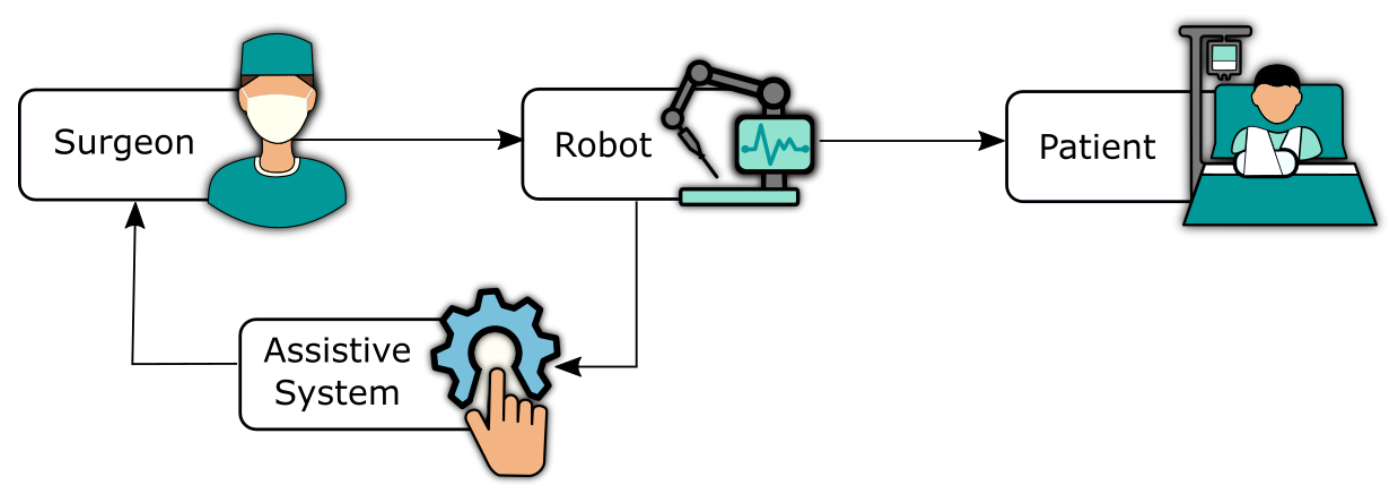

Fig. 3. Representation of a Level-1 system. The surgeon interacts with the robot which, in turn, provides the clinician with manual guidance or virtual fixtures. In this case, the control loop is closed by the surgeon, who has the full control of the machine for the whole duration of the procedure.

[Definition of Level 1] Systems classified within Level-1 "Robot Assistance" are capable of interacting with the surgeon to guide or support the execution of a particular task. The assistance provided consists in either active constraints to guide the surgeon's motion or virtual fixture to enhance the surgical site visualisation.

Defined as "Robot Assistance", this level includes platforms that provide some support to the operator/user, but never take control of the action being performed, as represented in Fig. 3. It is worth noting that, in the context of surgical robotics, the operator/user can always be identified with the surgeon in charge of the procedure, while the patient, often sedated, is the target. This is clearly different from other fields in medical robotics, such as rehabilitation, where the patient often plays the role of user and target at the same time.

The fundamental role of these technologies is detailed in the following section which is mainly focused on systems dedicated to supporting the surgeon in the execution of a specific surgical action. In our analysis, we first identify a number of enabling technologies that are crucial to achieve Level-1 autonomy. Then, we review approaches that provide passive 
assistance, producing information before and during robotic surgery and, thus, allowing a robot with Level-0 autonomy to reach Level-1. Examples include systems that suggest optimal robot deployment and ports placement, and systems providing augmented reality. We then analyse solutions that actively interact with the surgeon by providing mechanical support (i.e. guidance or compensation of periodic motion) and, lastly, we discuss the role of haptic feedback.

a)

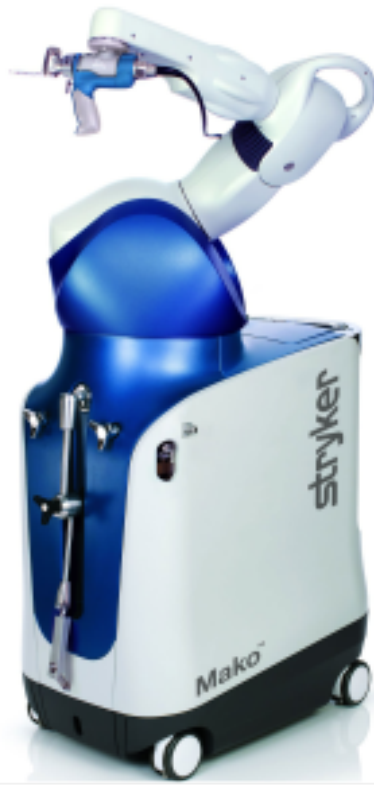

b)

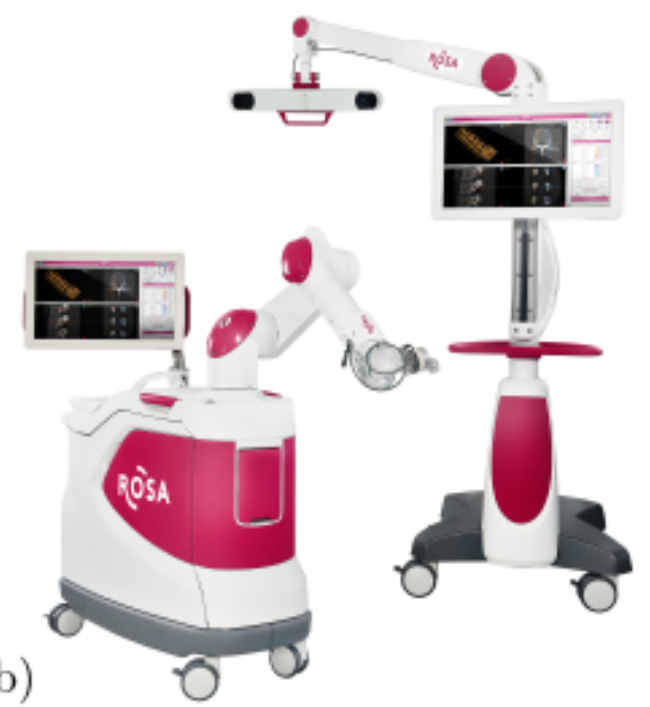

Fig. 4. The Mako from Stryker (a) and the ROSA platform by Zimmer Biomet Robotics (b) are used in orthopaedics for joint replacement.

\subsection{Enabling Technologies}

Most research platforms operating at Level-1 acquire a limited amount of information characterized by relatively low complexity, typically related to either the robot, the surgeon, or the target tissues. Therefore, tool tracking, eye tracking and tissue interaction sensing can be considered as three enabling technologies to achieve Level-1 autonomy. In addition, surgical robot controls paradigms are the foundation on which all the autonomy levels poses [16]. However, as the control specifications varies for every medical application, an in-depth analysis of control methods for surgical robotics is not discussed in this review.

\subsubsection{Tool Tracking}

Surgical tools tracking is a core component for developing assistive technologies such as augmented reality and haptic feedback. Approaches in literature can be clustered in three main groups. During the first half of 2000's, just computer vision was used [17]. Then, with the launch of surgical open research platform such as the daVinci Research Kit (dVRK) [18] and Raven II [19] and with the availability of kinematic data from the robot manipulators, new approaches were developed with improved robustness and lower dependency from light conditions [20,21]. More recently, machine and deep learning became popular, with papers using either Gaussian Mixture Models (GMM), Convolutional Neural Network (CNN) [22] and Random Forests [23]. Video stream and kinematic data were combined to strengthen the generalisation capabilities of machine learning models such as Random Trees [24]. A 3D-CNN structure was implemented to account for the correlation between subsequent frames in [25]. Ultrasound imaging was implemented in [26] to enhance accuracy.

Overall, works from the literature show that it is possible to track surgical tools at a rate of $29-30 \mathrm{~Hz}$ [22], thus guaranteeing a smooth real-time video stream, while maintaining a sub-mm tracking error for most applications.

\subsubsection{Eye tracking}

Eye-tracking is nowadays an established technology, commonly adopted in research fields outside surgery (e.g. customer behaviour and user experience). In robotic surgery, it can be used to capture the surgeon's gaze in augmented and mixed reality applications or to study surgical task recognition. 
In open or laparoscopic surgery the most common approach is the adoption of head-mounted devices (HMD). Such systems include glasses with tracking cameras [27] or optical trackers for estimating the pose of the head [28]. However, acceptance of HMDs by the surgical community is limited and a convincing clinical application, demonstrating effectiveness in a real surgical scenario, is still missing [29]. On the other hand, whenever a visualization device is already in use (i.e. surgical microscope, immersive user console), eye tracking is not disrupting the clinical workflow and can be easily adopted for guiding assistive tasks, such as instruments control by surgeon's gaze [30,31].

Overall, despite the availability of commercial systems for eye-tracking (e.g. Tobii Pro or EyeLink by Sr Research), translating this technology to surgical applications is not immediate as a strong case for its adoption has yet to be demonstrated via convincing clinical studies.

\subsubsection{Tissue Interaction Sensing}

A feedback on the interaction between instrument and tissue is crucial for safety and efficacy in both open and minimally invasive surgery. Research in providing surgical instruments with force/torque/grasping/contact sensing capabilities has been extensive in the last two decades $[32,33]$. However, this effort has been hampered by the additional complexity and cost that sensing adds to instruments that either need autoclave sterilization (if reusable) or extremely low fabrication costs (if disposable). For this reason, sensor-less options using force and torque estimation [34] or data-driven vision-based sensing [35] have recently gained traction in the research literature. More complex approaches adopt convex optimisation [36] or screw theory [37] to estimate tool dynamics. While extremely promising and straightforward to implement in commercially available Level-0 robotic platforms, these approaches have yet to be demonstrated outside a research lab environment [35].

\subsection{Passive Assistance}

In the context of this review, passive assistance technologies are intended as systems that assist the surgical activity by providing additional information to the surgeon. A considerable amount of work has been carried out in this field, particularly during the first decade of the century. Here, we focus on two specific research streams: assisted planning, relevant before the surgery starts, and augmented reality, which is available during the procedure to amplify surgeon's cognitive capabilities.

\subsubsection{Assisted Planning}

Optimal ports placement is a common issue in minimally invasive surgery (MIS) due to the limited reach, articulation and dexterity offered by endoscopic instruments. A poor placement at the beginning of the procedure may introduce undesirable delays and require re-placement while the patient is under anesthesia.

Laser pointers and light emitting diodes (LED) mounted on the tip of surgical tools were adopted in [38] to simplify the deployment of laparoscopic instruments. Other approaches capitalize on pre-operative analysis, such as computer tomography (CT) and magnetic resonance imaging (MRI), to develop virtual reality for planning in neurosurgery [39] or to minimize collisions in abdominal and thoracic surgery [40]. In orthopaedics, pre-operative 3D scans are used to manufacture patient-specific tool guides, thus increasing surgeons' accuracy during osteotomies [41]. All the mentioned contributions deal with hard tissues or instruments' geometry. Planning algorithms involving soft tissues is still an open challenge, due to the inherent complexities in modelling of the tissue and the lower reliability of registration with pre-operative imaging as reported in [42].

\subsubsection{Augmented Reality}

Introduced in surgery in 1986 [43], Augmented Reality (AR) gained momentum in the last three decades, enabled by the increased amount of computational power. With AR, additional information such as tumor location or hidden instruments can be shown to the clinician by superimposing virtual objects to the endoscopic image. Pre-operative images (CT, MRI, ultrasound) are used to extract the shape and location of the target. Subsequently, 3D models are registered to the anatomy. MRI- and CT-compatible fiducial markers can be adopted to address issues in registration [44]. Fluorescent fiducials are proposed to account for intra-operative deformations in [45]. The fusion of pre-operative annotated MRI and intra-operative trans-anal ultrasound is proposed in [46]. To provide high-level information in AR, context-awareness is required. An example is provided by [47] where different visualisations are proposed to the user depending on the tumor resection phase, autonomously detected by the system. The visualisation includes the targeted area, the resection margins or vital structures nearby the region of interest.

While most of the research target the surgeon as end user of the technology, [48] introduced ARssistant, an HMD that shows the location of robot instruments inside the patient's body to the assistant. This approach is particularly interesting from the clinical perspective, as literature reports many adverse events [49] caused by a lack of coordination between assistant and surgeon.

An exception worth mentioning is the success of AR for surgical training [50], with several commercially available platforms already in use [51], and clear potential for expanding training programs to low-resource settings around the world 
Overall, research in the field of AR for surgery and robotic surgery is well established. However, translation into clinical practice has not happened yet, possibly due to the lack of an objective metric to assess its impact and motivate adoption by the surgical community.

\subsection{Active Assistance}

Robotic systems actively interacting with the surgeon at Level-1 are classified as active assistance systems. These devices perform actions that affect the surgical procedure, such as applying forces to the user interface or restricting motion of the surgical instruments, based on a limited knowledge of the environment (i.e. force sensors, pre-computed forbidden areas, periodic inputs, etc.). In this case, the robot does not have the ability to control the execution of tasks, but rather reacts to actions initiated by the surgeon.

\subsubsection{Assistive Systems}

Minimally invasive surgery on soft tissues may be affected by periodic movements such as respiration or heart beat. Compensation of oscillatory motions has the potential of reducing undesired interaction with the anatomy. Techniques such as Smith predictors [53] and Fourier series models [54] were adopted for heart beating motion forecasting. Validation tests proved the system to be able to reduce by a factor five the tracking error of the system compared to state of the art. Experimental results reported in [55] show that such technology enhances the clinicians' dexterity reducing by a third the rate of missed hits in a suturing task.

In surgeries where the environment is more "stable" or better constrained, such as neurosurgery, microsurgery or orthopedic surgery, robot assistance can be provided to prevent undesired interactions with delicate structures. In this case, artificial repulsive force fields are generated and applied to the surgical tool tip. Passive control schema have been developed to guarantee the stability of the tool-tissue interaction [56].

In orthopedic surgery, active constraints are already part of commercial platforms, such as the Stryker Mako and the Zimmer Biomet ROSA, shown in Fig. 4, which improve precision during interventions such as knee cap replacement, total knee arthroplasty, or total hip replacement. On the other hand, in cardiology a first example of a commercial platform employed for automated surgery is proposed by Corindus Vascular Robotics which provide a "Rotate-on-Retraction" gesture to simplify the navigation of a guidewire within blood vessels [57].

\subsubsection{Haptic Feedback}

Surgeons heavily rely on tactile and force feedback during open surgery. Such feedback is severely hampered in MIS and completely lost in current robotic surgery. Robotics offers the opportunity to restore haptic sensation by means of sensors placed at the instrument and actuators integrated within the user interface. A large body of research exist in this field, mainly driven by technological advancements in sensing and actuation.

The most common approach is based on mechatronics [58], but pneumatic [59] and hydraulic [60] systems have also been proposed. Haptic feedback can be used for tissue palpation, thus identifying buried tissue structures or stiffer regions, to develop assisted guidance of surgical tools in a confined space [61], or to prevent instrument collision [62]. So far, the consensus from the surgical community has been that high definition 3D vision, combined with the high dexterity and precision of robotic tools, were sufficient to cope with the lack of haptic feedback [63]. Nevertheless, platforms such as the Transenterix Senhance [64], that recently entered the market, are equipped with haptic feedback. It will be extremely interesting to see if clinical data from surgeries performed with new robotic platforms will be convincing enough to modify the opinion of the surgical community.

\section{Level 2 - Task Autonomy}

[Level 2 of Autonomy] Systems classified within Level-2 "Task Autonomy" are capable of accomplishing specific surgical tasks upon specifications provided by the surgeon. The robot control switches from the human operator to the machine for the duration of the task to be executed. The second level of autonomy is defined as "Task Autonomy". At this level, the robot can take control of the procedure, but does not possess the ability to define any parameter for planning the task. The surgeon provides the information required to perform the action and the robot executes. The aim of task autonomy is to free the surgeon from the cognitive burden and/or fatigue associated to complex and/or repetitive tasks.

An example is tip retroflexion in magnetic colonoscopy. In colonoscopy, retrograde vision allows the operator to investigate a larger portion of the bowel. However, predicting how to change the controlling magnetic field and field gradient to achieve the desired motion at the tip of the endoscope is extremely complex for a human operator. In [65], an autonomous algorithm is proposed that tracks in real-time the pose of the endoscope tip and adjusts the pose of the external driving magnet 


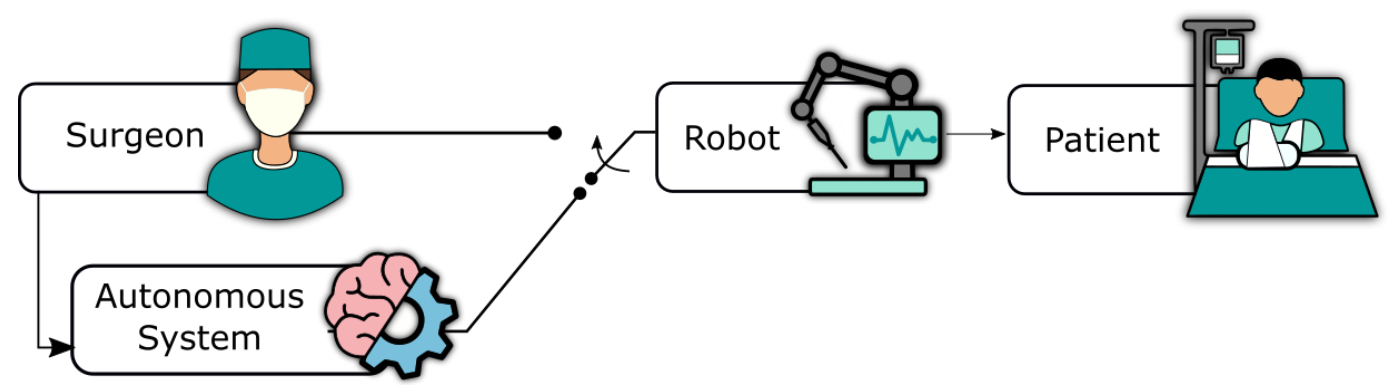

Fig. 5. In systems belonging to Level-2, the surgeon provides the necessary information for the robot to accomplish a given task. Since during the autonomous execution the control passes from the surgeon to the machine for the time needed to perform the action, we refer to this as discrete control, represented by the switch.

accordingly in order to achieve retroflexion. The robotic colonoscopy platform normally works in transparent teleoperation with active constraints (Level-1) and, when the operator needs retroflexion, the algorithm kicks in.

Similarly to retroflexion in endoscopy, tasks such as tissue retraction, suturing and ablation can be automated in robotic surgery. To enable these and other autonomous tasks execution at Level-2, technologies like task recognition and tissue palpation are essential. Papers discussing these lines of research are reviewed in this section.

From an ethical standpoint, task autonomy is the first level where the machine takes full control of the surgical instruments, although for specific gestures and under the supervision of the surgeon. This "discrete shared control" introduces a paradigm shift in the ethical and regulatory framework that needs to be addressed by notified bodies and surgical robotics companies alike to allow autonomy to get into the operating rooms. This topic is discussed in more detail in Section 7.

\subsection{Enabling Technologies}

At Level-2, the robot does not own the ability to elaborate decisions; nevertheless, it is required to retrieve information with higher complexity with respect to Level-1. For this reason, we selected a technology that has a great impact on systems at this level: Gesture Classification. Gesture Classification can enhance the ability to activate the robot at the right time and minimize the disruption to the surgical workflow. By means of gesture classification, the robot is capable to follow the clinician's work plan, thus providing dedicated support depending on the phase of the operation.

\subsubsection{Gesture Classification}

A correct surgeon-assistant interaction is crucial to reduce the chance of mistakes during surgery [49]. In Level-2 the robot can be considered as an assistant, executing basic sub-tasks. To achieve a satisfactory coordination between surgeon and robot, the identification of the surgical task is crucial. To achieve this, several solutions have been proposed using source of information such as endoscopic videos [66], real [67] and simulated kinematic data [68] and depth images [69]. Video streams and kinematic data can introduce significant computational burden, preventing the system to work in realtime. To tackle this issue, machine learning models such as Hidden Markov Models [70], weakly supervised Gaussian Mixture Model [67], multiple kernel learning [66] and Recurrent Neural Networks [71] have been adopted. Promising results have been achieved on bench-top test scenarios. However, as the complexity and variability of real tasks increases, the model accuracy, generally ranging between $70 \%$ and $85 \%$, tends to decrease, thus limiting applicability in their current implementations.

\subsection{Suturing}

Although widely performed in many surgeries, suturing remains a critical task as failures might lead to disastrous consequences. Surgeon ability and experience play a crucial role in the quality of a suture, thus automating this repetitive task would guarantee more uniform outcomes and relax the cognitive burden on the surgeon.

The execution of autonomous suturing is generally divided in two stages: the insertion of the needle, during which the needle pierces the tissue and is re-grasped at the exit point, and the tying of a knot to secure the suture with a surgical thread. A significant amount of literature regarding each sub-task is available.

\subsubsection{Needle Insertion}

The needle insertion stage entails high precision in estimating the optimal position, angle and applied force required to pierce the tissue. Moreover, the discontinuous grasp of the needle generates uncertainties in the pose estimation. Finally, as the needle pushes through the surface, the tissue is subject to deformation, thus increasing the needle pose uncertainty. For 
these reasons, autonomous needle insertion raises interesting technical challenges, mainly related to the needle geometrical model and tissue deformation. A combination of kinematic and geometric modelling is proposed in [72], where the trajectory is generated to minimize the tissue deformation. Estimation approaches such as Unscented Kalman Filter [73] and an online evaluated deformation matrix [74] were used to estimate the tissue and needle deformation.

A crucial aspect of needle insertion is the definition of the entry points. In order to simplify this problem, optical markers [75] and laser pointers [76] were integrated with optimisation techniques, with the aim of minimising the tissue strain. However, the intra-operative placement of optical markers could be undesirable in surgery, thus reducing the advantages of autonomous execution. [77] proposed a solution for a single-master dual-slave platform for semi-autonomous needle insertion. The surgeon controls one arm to insert the needle while the second arm, triggered by the insertion force, collects the needle and returns it to the surgeon.

More advanced approaches improve the success rate by adopting transfer learning (a method to transfer the learnt knowledge from an artificial intelligence model to address a different problem) [78], which reports a success rate of $87 \%$ in needle driving, or Sequential Convex Programming [79].

Even though satisfying results are reported on bench-top trials, validation on a realistic scenario considering tissuespecific mechanical properties and the presence of anatomical structure at risk, such as nerves and blood vessels, is still missing.

\subsubsection{Knot Tying}

The last step in suturing consists of tying a knot .The main technical challenge is related to the deformability and resistance of the thread, which could lead to undesired entanglement, thus damaging the tissue. The complexity of the task is further increased by limited dexterity, confined workspace and lack of tactile feedback. To mitigate uncertainties on the thread deformation, [80] proposed to apply a constant tension. The paper shows the feasibility of two different knots with performance comparable to human execution (nearly 10 seconds). Interestingly, during retraction, the tissue is subjected to external forces and deformation, thus requiring a continuous re-planning. More advanced techniques enhance the robustness of the autonomous system by using machine learning approaches such as Learning by Observation (LbO, also known as Learning by Demonstration) to extract the fundamental features from human gestures. In [81], manually performed tasks are used to train Long Short Term Memory Recurrent Neural Networks. This type of neural network is particularly interesting as it is capable of considering temporal evolution of features, thus allowing the algorithm to learn complex sequences of gestures typical of knot tying. In [82], $\mathrm{LbO}$ is used to generate trajectories on a phantom starting from recorded manual sutures, achieving an accuracy of $2 \mathrm{~mm}$ in the path execution.

As the methods proposed in the literature vary significantly, an objective comparative assessment of the performance is not straightforward. For this reason, [83] proposed an evaluation metrics, comparing 4 different approaches. However, thread deformation still hinders satisfactory results. The adoption of high visibility threads may simplify the tracking problem, reducing the uncertainty on the pose detection of the string.

\subsubsection{Supervised Suturing}

Literature that simultaneously tackle needle insertion and knot-tying on commercial robotic systems is limited. The most convincing solution at the moment entails the development of a dedicated platform, the Smart Tissue Autonomous Robot (STAR), for full autonomous anastomosis [84]. The system is composed of a 7-DOF KUKA LBR arm equipped with a custom suturing tool [85] (Figure 6). Two working modes are available: in automatic mode, the system autonomously evaluates the position of each entry point, starting from the suturing outline defined by the surgeon, in manual mode each entry point is defined manually. Tests on phantoms demonstrated that the system is capable of completing a suturing task 5 times faster than a robot-assisted procedure and 9 times faster than an operator. It is worth to point out that, in both working modalities, the surgeon is required to define the suturing profile of the anastomosis. Systems capable of autonomously retrieving the suturing task specifications will be introduced in Section 5.3, at Level-3 of autonomy.

\subsection{Tissue Retraction}

During MIS procedures, a significant amount of time is spent mobilising and dissecting tissue to reach the area of interest. In this context, dissected tissue is often retracted to expose the surrounding area. Although this gesture is performed frequently and, thus, would make a good candidate for task automation, few contributions are available in literature. This may be due to the complexity associated with detecting and tracking deformable soft tissue during surgery. Simulation frameworks have been developed in $[86,87]$ to plan a grasp-and-retract gesture. The strategy aims at minimising the tissue strain, simultaneously avoiding tearing and guaranteeing an obstacle-free trajectory. Recent studies $[88,89]$ present tissue retraction on a dVRK involving visual markers to identify the flap grasping point and fuzzy logic to execute the gesture. Despite the promising results, only bench-top experiments are available. In a real scenario, the complexity of tissue detection may be significantly higher, considering the tissue elasticity and the presence of tools. For this reason, automating tissue 

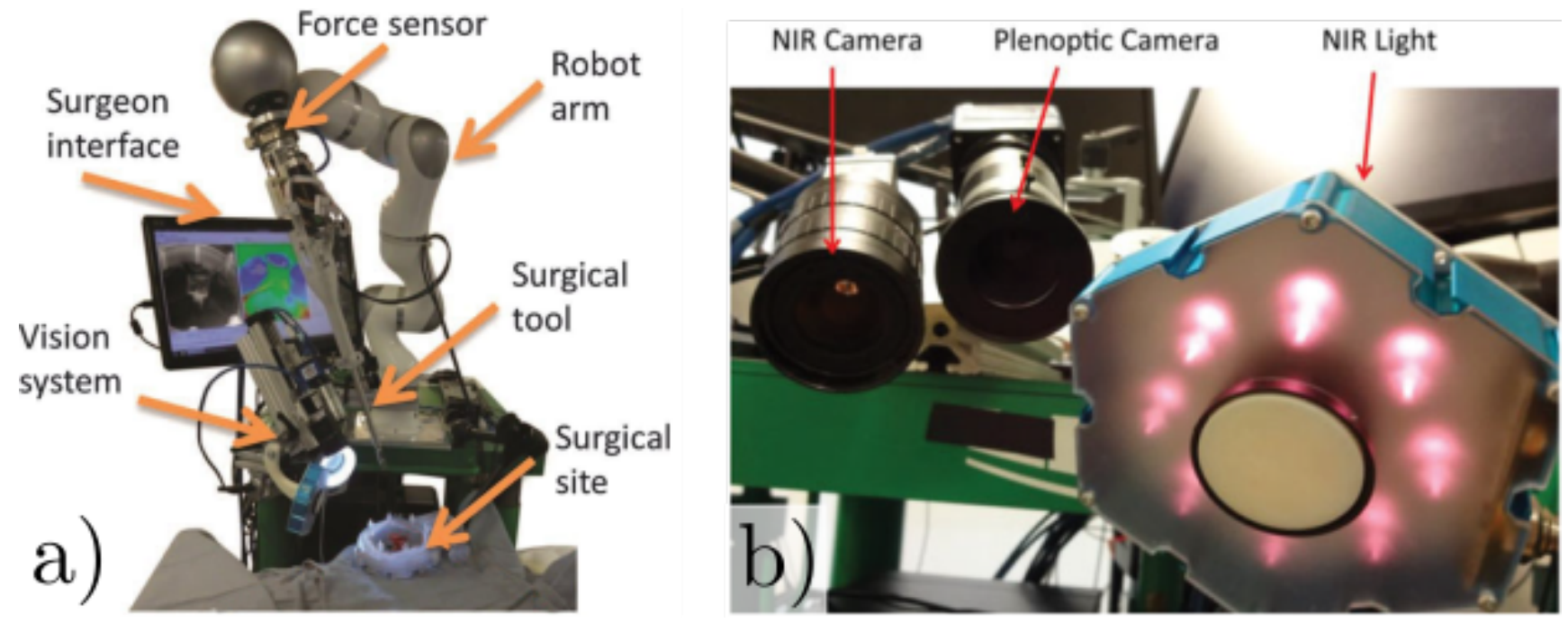

Fig. 6. The Smart Tissue Anastomosis Robot (STAR) system (a). The system is equipped with Plenoptic camera to retrieve depth information while the Near Infra-Red (NIR) camera detects hidden structures in the tissue (b).

retraction remains an open challenge.

\subsection{Stiffness Mapping}

Manual palpation is commonly used in conventional surgery to identify and dissect malignant masses below the surface of organs (i.e. kidneys, lungs). In robotic surgery, the lack of tactile feedback hinders the surgeons' ability to evaluate the tissue properties. Haptics, discussed in 3.3.2, aims at restoring this ability. A further step towards the execution of autonomous tasks such ad dissection and ablation (see Section 4.5) is the ability to autonomously estimate the tissue properties by mechanical contact. To provide palpation, array sensors based on different principles such as the measure of distributed pressure on a surface [90] and the Bernoulli pipe structure [91] have been adopted, detecting hard inclusions with a precision of $97 \%$. Hall sensors were implemented on a daVinci instrument tip [92] to localise blood vessels to a maximum depth of $5 \mathrm{~mm}$. A different approach for prostate palpation [93] is based on the adoption of an attachable sensor matrix. Approaches based on sensors are limited by increased complexity, cost and sterilization requirements. In a seminal paper, [94] demonstrate sensorless palpation with a multi-backbone continuum robots for the first time. Based on this work, [95] proposes a smart navigation approach supported by pre-operative images.

In [96], elastography had been used to collect a dataset and develop a machine learning model for autonomous detection of hard inclusions in a phantom. Most of the proposed strategies for palpation adopt custom, hand-held instruments, thus increasing the number of surgical accesses required. A solution integrated with commercial systems would be preferred. Moreover, the adoption of dedicated mechanical devices introduces complexities associated to reprocessing, possible contamination and production costs, thus significantly limiting the potential for clinical translation.

\subsection{Ablation}

Ablation consists in eradicating a portion of compromised tissue by transferring a high amount of energy to the target means of electric cauterizers, cryoprobes or High Intensity Focused Ultrasound (HIFU). The major risk is undesired removal of healthy tissue from surrounding structures such as blood vessels or nerves bundles. The correct localization of the target tissue to remove and the precise identification of its margins pose technical challenges, especially in surgical excision of cancer, where tumors may be concealed underneath healthy tissue. In these procedures, it is also crucial to spare as much healthy tissue as possible to prevent organ failure and subsequent need of a transplant, should this be an available option. A possible approach is to perform mechanical palpation to create a local stiffness map for guiding ablation [97, 98], as discussed in Section 4.4. Alternative imaging methods such as ultrasound [99] and optical coherence tomography [100] may be adopted to guide cardiac ablation.

Following a common practice in surgery, several works considered laser ablation to reduce direct interaction with the anatomy [101-103]. Nonetheless, the lack of physical contact complicates the identification of the target area, which is manually selected by the surgeon before starting the procedure [102]. To relax the input required from the surgeon and increase the autonomy in detecting the target, preoperative scanning and voxel-growing on the 3D anatomic model were successfully implemented [101].

In order to avoid heat generation [104] adopted cryoprobes, while [105] used pressurized water jets. The latter is a com- 
mercially available system (branded as aquabeam) designed for the treatment of benign prostatic hyperplasia. Although the prostate profile is identified by the surgeon, on ultrasound scan, the resection is autonomously performed by a high-pressure water jet. This is a remarkable example of a Level-2 system reaching the operating room, enabled by the simultaneous use of intra-operative ultrasound imaging and robotics. Extending Level-2 systems for autonomous ablation to other surgical procedures will be challenging whenever the localisation of the target area is hindered either by tissue deformation or lack of visualization.

\section{Level 3 - Conditional Autonomy}

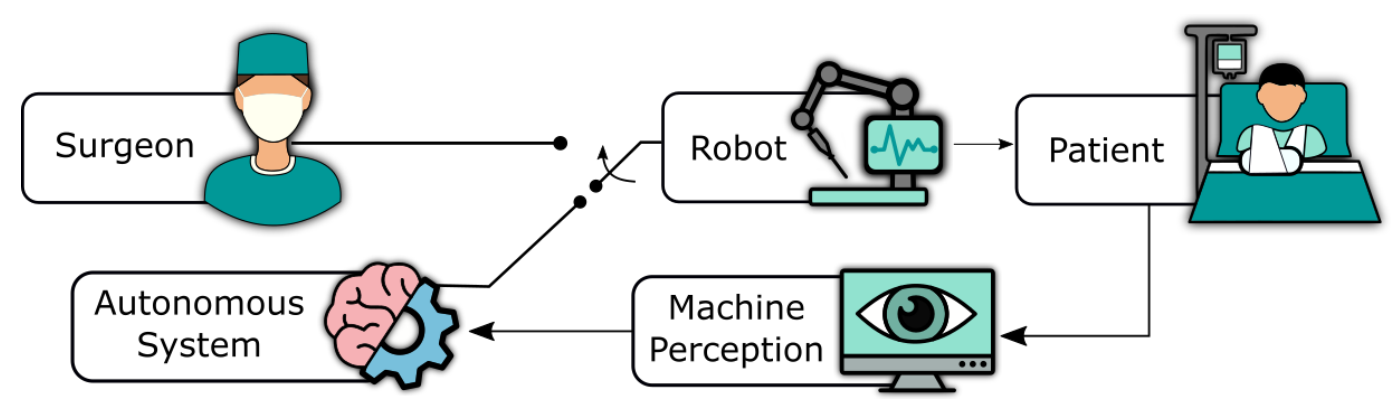

Fig. 7. Systems belonging to Level-3 are capable of autonomously define the specifications to plan and execute a surgical task, differently from Level-2 systems where the surgeon was supposed to provide them to the system. Similarly to Level-2, discrete control takes place, as represented by the switch.

[Level 3 of Autonomy] Systems categorised in Level-3 "Conditional Autonomy" are provided with perceptual capabilities to understand the surgical scenario, to plan and execute a specific task and to update the plan during execution. Similarly to Level-2, robot control switches from the surgeon to the machine for the duration of the task to be executed. The main characteristic of Level-3, defined as "Conditional Autonomy', is the ability to conceive strategies to perform a specific task, always relying on the human operator to approve the most suitable to be implemented. In the context of robotic surgery, this reflects the ability to autonomously extract the parameters required to plan a specific task from the information available to the system. During task execution, the environment is constantly monitored and the plan is updated in real-time. In case of performing a suturing task at Level-3, for example, the system would be able to extract the suturing points and the length of each suture from real-time imaging, then plan and execute the suture autonomously. Real-time imaging would also provide continuous updates to the plan as the task is performed. This example is discussed in detail in Section 5.3. Other examples are autonomous navigation of flexible endoscopic robots in unstructured environments [106], autonomous navigation in the abdominal anatomy, and autonomous anastomosis.

\subsection{Enabling Technologies}

At Level-3, the system requires the ability to perceive, extract and analyze contextual elements to plan how to execute a task and to update the plan during execution. Similarly to the surgeon's cognitive process, systems at Level-3 are expected to extract high-level features from the surgical scenario and to act upon them in real-time. Key elements to achieve this are computationally-efficient tissue models, advanced imaging capabilities, and algorithms to track high-level features in the environment.

A significant help in this context comes from the giant leap in computational power of graphic processing units (GPUs) that we have experience in the last decade. Current GPUs, mainly developed for the gaming industry, can be used to run complex algorithms at an unprecedented speed.

\subsubsection{Tissue Modelling}

Predicting tissue deformation plays a crucial role in manipulating soft tissues. Furthermore, understanding the mechanical properties is essential to avoid unintentional damages. Research has focused on deformation assessment by means of data-driven approaches in needle insertion [107], and real-time detection of tissue perforation during spine interventions [108]. Intra-operative real-time images [109] and pre-operatory CT scans [110] were used to obtain anatomy-specific deformation models. However, one of the main challenges is the intra-operative real-time identification of tissue parameters describing the elasticity and stiffness of a given surface. Recently, 3D displacements and kinematic data were combined to evaluate the deformation through optimization techniques in $[111,112]$. Results demonstrated the system ability to evaluate 
the parameters in real-time, but quantitative assessments of the performances are not available. Only one work presented an approach to model the cardiac atrium for guided manual ablation with a Stereotaxis platform [113] with successful intraoperative results.

\subsubsection{Advanced Imaging}

Real-time feature extraction from sensing sources is crucial for the automation of surgical tasks. Even with state-of-theart white light stereoscopic imaging, it is still a major challenge to have reliable online understanding of the surgical scene. For this reason, a number of advanced imaging approaches have been proposed, including "plenoptic" vision to retrieve depth from the scene. Plenoptic cameras are equipped with a micro-lens array capable of acquiring different points of view of the same scene in a single acquisition, thus allowing an accurate 3D reconstruction [114]. Tridimensional reconstruction accuracy is strengthen by the possibility of perceiving the light direction by this type of cameras. If combined with fluoroscopy imaging as in [114], plenoptic cameras allow to identify internal hidden structures such as blood vessels and nerves. Although a small amount of contributions is available in literature, interesting results have been obtained so far, such as the completion of a needle insertion [115], Level-3 suturing (as detailed in Section 5.3), and vitreoretinal surgery [116]. Alternative imaging technologies currently under evaluation to enhance feature extraction are hyperspectral imaging [117] and TeraHertz vision [118].

Considering the novelty of the field and the promising results achieved, future developments of these technologies will play a crucial role in surgical robotic research.

\subsubsection{High Level Feature Tracking}

Differently from the tracking discussed in Section 3.1.1, where the surgical tool was intended as a physical extension of the robotic platform, here we focus on tracking of tools or features that are physically disconnected from the surgical robot.

In the context of suturing, moving from Level-2 to Level-3 without taking advantage of a dedicated tool, as in the work discussed in Section 4.2.3, requires the ability to track the suturing needle and thread throughout the execution of the task. In the field of suturing thread detection a combination of color and geometry segmentation [119] are adopted to detect and model the thread as a spline. However, given the thin structure of a suturing thread, basic computer vision algorithm may suffer critical loss of performances in a real scenario where the light condition is insufficient and the environment is cluttered. For this reason, data-driven analysis of images to retrieve the 3D pose of the thread [120], image-based optimization techniques [121] and Markov Random Fields-based solutions [122] are presented to reject such disturbances.

In the context of suturing needle tracking, an effective approach consists of equipping the needle with highly visible markers [123], detectable by conventional white-light cameras. In [79], the detection by means of coloured markers is supported by a custom gripper that reduces the needle mobility.

Another interesting tracking problem is related to reconstructing in real-time the shape of biopsy or injection needles under ultrasound guidance. To address this challenge, motion features are explored in [124], while a Kalman filter is proposed in [125]. Optimization techniques based on gradient descent algorithms are adopted in [126] along with geometric needle models for tracking. Although the results of [124] reports a localization accuracy of $1.70 \mathrm{~mm}$ while respecting the real-time constraint [121], there are no contributions addressing the problem of high level feature tracking in a realistic scenario, thus motivating further investigation in the field.

\subsection{Navigation of Continuum Surgical Devices}

Continuum surgical devices include, among others, steerable needles for biopsy sampling or local drug delivery and cardiovascular catheters. In this section, we discus robotic platforms pursuing Level-3 navigation of these types of devices.

Robotically controlled needles may introduce a relevant benefit in brain, prostate and lung surgery, where the difficult access to the anatomy increases the complexity of the task. Due to their thin structures and tortuous paths, the manual navigation of these needles is demanding, if not impossible. To enable an effective use of these devices, autonomous navigation is crucial and continuous updates of the external forces acting on the needle are required to safely navigate towards the target. Moreover, as the needle pushes through, the system must compensate the tissue deformation to avoid undesired interaction with peripheral anatomical structures. [127] proposes a kinematic and a mechanics-based approach to evaluate needle-tissue interaction, thus predicting tissue deformation. A crucial aspect of steerable needles is localisation and registration to the anatomy. Ultrasound imaging is widely adopted [128] to develop image-based control strategies: as the needle advances through the tissue, an ultrasound transducer tracks and follows the tip. Alternative imaging approaches used for autonomous needle navigation include intra-operative MRI to localise and avoid obstacles [129] and Fiber Bragg Grating to track the needle tip [130]. Robotic needle guidance is a relatively new approach in robotic surgery, therefore no currently available clinical platform embeds this technology. However, promising results have been recently obtained in human cadaver trials [131] and with the support of preoperative analysis [132], demonstrating a possible translation to Level-4 autonomy in the near future. 
In the context of autonomous navigation of cardiovascular catheters, a very advanced work is presented in [133], where force sensing and palpation are adopted to drive an autonomous catheter through blood vessels, up to the heart. The approach is validated by an in-vivo trial, demonstrating performances comparable to the manual execution.

\subsection{Advanced Suturing}

In order to achieve Level-3 suturing, plenoptic cameras have been adopted to extract the 3D profile of the scene and autonomously define the suture entry points in [115]. The algorithm is based on human demonstrations and validated on ex-vivo tissues, showing a superior performance in terms of time and accuracy when compared to a human operator. Point clouds were used in [134] to autonomously plan the needle path, including the entry points. In particular, the region of interest is identified manually by the surgeon to reduce the computational burden, but then the system takes over by extracting all the task specifications autonomously. The system is evaluated on a suturing phantom by assessing the thread tension and the displacement of the entry points. Results show a consistency almost three times higher than a human operator. While current results on advanced suturing are extremely encouraging, they are limited to anatomical phantoms or ex-vivo tissue models. As the approach is translated to a more realistic scenario, the performance of the suturing robot may be heavily affected. From the small amount of literature available, it is clear that full autonomous suturing is still far from being commercially available. Moreover, due to the high complexity of the task, no studies addressing the problem of failure modes, such as the accidental drop of the needle or the entanglement of the thread, have been carried out. Embedding the technologies included in Section 5.1.3 could potentially revolutionise the approach to autonomous suturing by providing a robust and continuous tracking of needle and thread, thus allowing the system to consider their presence in the scene.

\section{Level 4 - High autonomy}

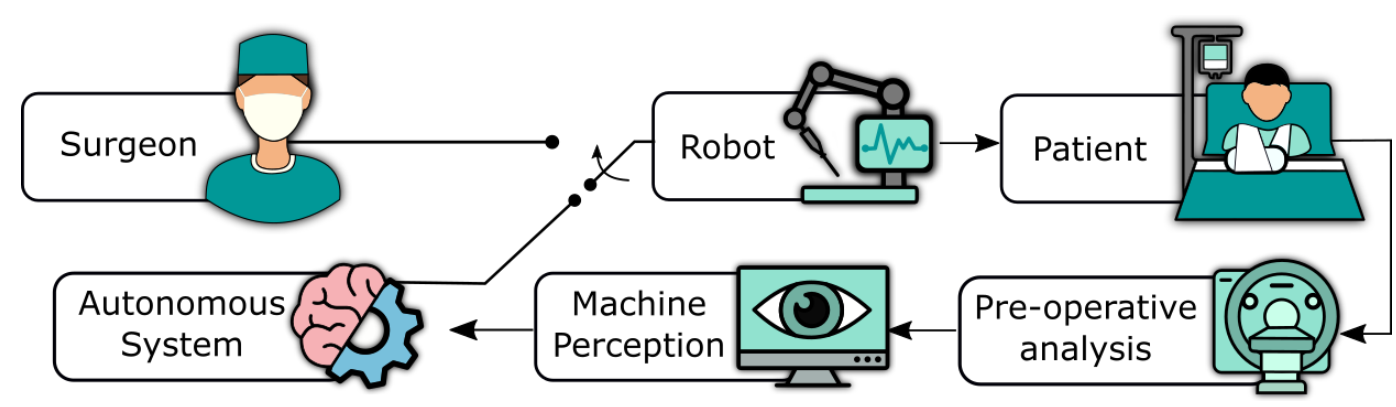

Fig. 8. In "High Autonomy" systems, pre-operative and intra-operative information are used to devise an interventional plan composed by a sequence of tasks, and execute it autonomously, re-planning if necessary. A surgeon always supervises the procedure and can get back control at any time.

[Level 4 of Autonomy] Surgical systems at Level-4 "High Autonomy" can interpret pre-operative and intra-operative information, devise an interventional plan composed by a sequence of tasks, and execute it autonomously, re-planning if necessary. Also in this case, a surgeon supervises the system under the discrete control paradigm. The fundamental characteristic of Level-4 systems is their ability to autonomously make clinical decisions and execute them, under constant supervision by the surgeon.

Beyond clear technical challenges, this level poses very relevant issues in terms of ethical and regulatory aspects.

While concrete examples are not yet available, we can easily see where these systems would clearly contribute to healthcare delivery, i.e. intelligent removal of cancerous tissue, from registration with pre-operative imaging, adaptation of the plan with real-time data, and ablation of cancer while maximising sparing of healthy tissue.

In this Section, we discuss how progresses in organ and tumor segmentation represent a stepping stone towards debridement and tumor resection. This section is then followed by a discussion of ethical and regulatory issues around autonomy in sugical robotics.

\subsection{Enabling Technologies}

\subsubsection{Organ and Tumor Segmentation}

Interpretation of pre-operative imaging (MRI, CT and US) is a requisite for a Level-4 system. Autonomous segmentation of organs such as brain [135], liver [136] and prostate [137] were investigated using different imaging techniques including 
CT [138], MRI [137]. Tumor profiles can be extracted from CT scans by adopting optimization techniques [137] and deep learning models $[136,138]$. Subsequently, the extracted regions are merged together to obtain a 3D model of the target.

Autonomous segmentation techniques are embedded in commercially available systems such as the Brainlab iPlan [139], which is capable of segmenting MRI scans and integrating them with other imaging techniques like ultrasound and elastography. Using this platform, the surgeon can validate the software segmentation, plan and deliver radiotherapy by means of robotic platforms, i.e. GammaKnife and CyberKnife. Although already implemented and used in the operating room for neurosurgery, autonomous segmentation remains an open challenge for thoracic and abdominal surgery, where tissue deformability prevents satisfactory results to be achieved.

Timeline of an Autonomous Tumor Resection

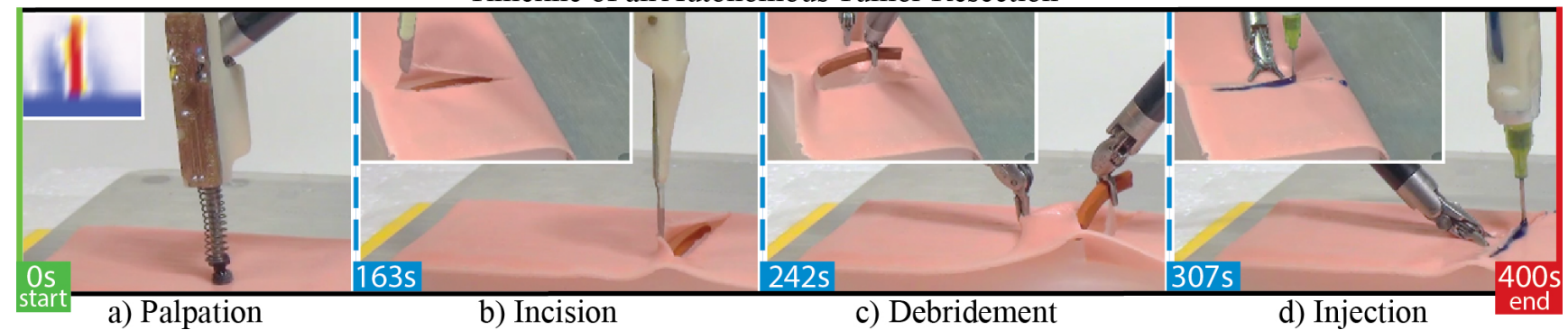

Fig. 9. Stages of autonomous tumor debridement on a phantom reported in [92]. Initially, a palpation probe scans the tissue to define the tumor boundaries (a). Subsequently, an incision is performed (b) and the tumor removed (c). Eventually, the incision is sealed by means of surgical glue.

\subsection{Debridement and Tumor Resection}

To perform tumor resection, surgeons are required to fuse pre-operative (e.g. MRI, CT, US) with intra-operative (e.g. white-light endoscopy, fluoroscopy, Near Infrared (NIRF)) imaging modalities. Systems with "High Autonomy" must possess a similar ability. In [140], NIRF markers were adopted to retrieve the tumor boundaries and guide resection via electrosurgery, while maintaining a minimum margin of 4-mm. Despite the markers were applied manually, this shows the feasibility of a NIRF-based visual servoing for debridement on phantom, achieving a margin of $3.67 \pm 0.89 \mathrm{~mm}$. A similar approach is presented in [141], where the target area is detected by applying coloured markers on a phantom. The debridement is modelled by means of Finite State Machines (FSM), allowing a gesture execution time of 20.8 seconds. Alternatively, a palpation probe [92] mounted on a daVinci manipulator was used to identify and excise a tumor hidden below the tissue surface (Figure 9. Subsequently, the wound was sealed with surgical glue [142]. The probe initially scanned the area of interest, localising hard inclusions in the tissue and tracking the tumor profile. Then, a surgical scalpel performed the incision and grasped the tumor. Experimental results on phantoms showed a success rate of 50\% in tumor excision. Despite the preliminary results, the system addressed some of the major challenges in surgical autonomy at Level-4 and did not require any intervention from the operator.

\section{Legal and ethical aspects of autonomous surgical robots}

The taxonomy and definition of the levels of autonomy are directly inspired by the SAE J3016 [2] standard, which defines the same levels for on-road Autonomous Vehicles (AV). Despite the standard was published in January 2014, the first examples of semi-autonomous cars date back to the 1970's. The sector experienced great advancements in the last two decades with the support of initiatives like the DARPA Grand Challenge, attracting interest from private companies. The recent technical and regulatory advancements have been massive, to the point that the US department of transportation issued a document entitled "Ensuring American Leadership in Automated Vehicle Technologies" and pilot trials of Level-4 and above have started on public roads in US, Canada and Europe. In the context of surgical robotics, the first (baby) steps to move autonomous platforms (Level-3 or higher) out of university labs are in the military field, with the US department of defense issuing a call for autonomous systems in combat zones [143]. However, in less extreme frameworks, ethical concerns arise regarding the consequences of decision errors and incorrect robot behaviours, potentially leading to serious injuries or even death. Being such a novel and fast-paced field of research, literature on this topic is scarce and mostly speculative. An interesting perspective is given by [144], in which three elements of responsibility are highlighted: Accountability, Liability and culpability. The first element is related to the ability of explaining decisions, which decreases as the system complexity increases and could be addressed by a combination of explainable AI and recording black boxes, similarly to aircraft. The 
element of liability, much discussed also for $\mathrm{AVs}$, could be addressed by insurance coverage or alternative approaches, like the concept of electronic personhood, introduced by the European resolution of 16 February 2017 [145]. On the topic of liability, other documents issued by the EU discusses issues related to AI and robotics [146]. Finally, culpability (i.e. the possibility of punishing) constitutes the most complex topic, and could pose a significant legal and ethical barrier, having influence on the surgeons' role.

An interesting contribution [147] focuses on the ethical aspects of autopsies, concluding that explainable AI and machine learning could give powerful support to forensic analysis only in a context of human-robot collaboration.

On the topic of patients' perspective, initiatives like the iRobotSurgeon survey [148] tries to assess the public acceptance of autonomous surgical robots, while [149] discusses the issues related to privacy, suggesting to adopt the "contextual integrity" theory.

From the regulatory perspective, notified bodies such as the American FDA, the British Medicine and Healthcare Regulatory Agency (MHRA) and the German Federal Institute for Drugs and Medical Devices (BfArM, in German) do not have specific frameworks for autonomous robots. The FDA currently classifies surgical robots as Class-IIb devices, while implantable and self-activating devices like peacemakers (which have some degree of autonomy, not requiring any human intervention) belong to Class III. One reason for the current classification of robots is the absence of autonomy. On the other hand, Class-III devices are limited to low-complexity and simple functional mechanisms in which the failure modes and operating conditions can be evaluated extensively, thus performing a complete risk evaluation, as required by all the medical devices standards (e.g. ISO 13485, 14791, 62304). Moreover, different approval pathways for Class-II and Class-III devices require significantly increased investment, as thoroughly described in [150]. As discussed in [151], at low autonomy levels, the current legal frameworks could be suitable to evaluate new devices. A common approach to introduce autonomy is to leave the surgeon in charge of activating the autonomous features. Despite being simple, this method greatly limits the effectiveness of the devices.

The use of machine learning algorithms also presents several issues in current regulatory schemes. All the medical devices standards prescribe a development process based on risk evaluation and minimization, but modern deep-learning approaches treat information in such a way to prevent a detailed risk analysis. State-of-the art approaches based on the novel topic of explainable AI could solve this issue.

At high autonomy levels, the robotic systems are supposed to make clinically-relevant decisions. This could introduce another regulatory dilemma: notified bodies like FDA lack the legal authority to regulate medicine, as this practice is usually left to medical societies. The latter, on the other hand, lack the technical competence to dominate complex and continuously evolving technologies such as robotics. A possible solution is proposed in [152], suggesting to include ethical elements in the engineering development process from the very beginning.

\section{Conclusions}

The development of intelligent machines will be a long and difficult endeavor, marked by a number of incremental steps in which science and technology will drive changes in societal behavior and legislative framework. This is particularly evident in medicine, where novel solutions motivate regulatory changes and societal perception of how healthcare should be offered. Robotic surgery is no exception and the success of the daVinci surgical system demonstrates the gradual acceptance, and now the preference, of new robotic technology with respect to traditional surgical approaches. The addition of reasoning capabilities to surgical robots will require some time, primarily because of the many open regulatory and liability issues.

However, if clear benefits are demonstrated, patients will accept and eventually demand devices that can provide additional cognitive and physical support to the surgeons.

This paper aimed at providing a first comprehensive mapping of levels of autonomy that could eventually be added to surgical robots, their implementation through enabling technologies, and the translation of these abstract concepts into practical clinical examples (Figure 1). So far, only few laboratory experiments have shown a clear advantage of autonomy in surgical robotics when compared to conventional approaches, and no clinical evidence exists yet. However, if research keeps the current pace, positive evidence will soon emerge and build up to an extent that will motivate notified bodies and hospital's ethical committees to consider transition to clinical trials.

This progress will require unprecedented levels of collaboration among engineers, surgeons and healthcare operators to ensure that communication among all actors in the operating theater is improved by the new technology. Human-machine interaction will be a key factor for the success of autonomy in surgical robotics. Only platforms that possess an effective way to communicate their intent and "explain" their decision to their human companions will find their way into the operating room of the future.

\section{Acknowledgements}

This work was supported by the Royal Society, by the Engineering and Physical Sciences Research Council (EPSRC) under grant number EP/R045291/1, and by the European Research Council (ERC) under the European Union's Horizon 2020 
research and innovation programme (grant agreements No 742671 and No 818045). Any opinions, findings and conclusions, or recommendations expressed in this article are those of the authors and do not necessarily reflect the views of the Royal Society, EPSRC, or the ERC.

\section{References}

[1] Yang, G.-Z., Cambias, J., Cleary, K., Daimler, E., Drake, J., Dupont, P. E., Hata, N., Kazanzides, P., Martel, S., Patel, R. V., Santos, V. J., and Taylor, R. H., 2017. "Medical robotics-Regulatory, ethical, and legal considerations for increasing levels of autonomy". Science Robotics, 2(4), p. eaam8638.

[2] SAEJ3016 201806, 2018. Taxonomy and Definitions for Terms Related to Driving Automation Systems for On-Road Motor Vehicles. Standard, SAE International, Warrendale, Pensylvenia, US, June.

[3] Simaan, N., Yasin, R. M., and Wang, L., 2018. "Medical Technologies and Challenges of Robot-Assisted Minimally Invasive Intervention and Diagnostics". Annual Review of Control, Robotics, and Autonomous Systems, 1(1), may, pp. 465-490.

[4] Hoeckelmann, M., Rudas, I. J., Fiorini, P., Kirchner, F., and Haidegger, T., 2015. "Current capabilities and development potential in surgical robotics". International Journal of Advanced Robotic Systems, 12.

[5] Nelson, B. J., Kaliakatsos, I. K., and Abbott, J. J., 2010. "Microrobots for Minimally Invasive Medicine”. Annual Review of Biomedical Engineering, 12(1), pp. 55-85.

[6] Bergeles, C., and Yang, G. Z., 2014. "From passive tool holders to microsurgeons: Safer, smaller, smarter surgical robots". IEEE Transactions on Biomedical Engineering, 61(5), pp. 1565-1576.

[7] Vitiello, V., Su-Lin Lee, Cundy, T. P., and Guang-Zhong Yang, 2013. "Emerging Robotic Platforms for Minimally Invasive Surgery”. IEEE Reviews in Biomedical Engineering, 6(1), pp. 111-126.

[8] Dahroug, B., Tamadazte, B., Tavernier, L., Weber, S., and Andreff, N., 2018. "Review on Otological Robotic Systems: Toward Micro-Robot Assisted Cholesteatoma Surgery". IEEE Reviews in Biomedical Engineering, XXX(XXX), pp. 1-19.

[9] Smith, J. A., Jivraj, J., Wong, R., and Yang, V., 2016. "30 Years of Neurosurgical Robots: Review and Trends for Manipulators and Associated Navigational Systems". Annals of Biomedical Engineering, 44(4), apr, pp. 836-846.

[10] Faria, C., Erlhagen, W., Rito, M., De Momi, E., Ferrigno, G., and Bicho, E., 2015. "Review of Robotic Technology for Stereotactic Neurosurgery". IEEE Reviews in Biomedical Engineering, 8, pp. 125-137.

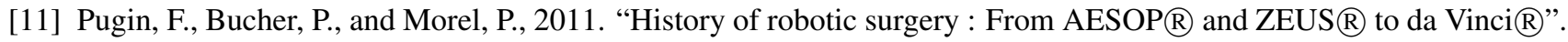
Journal of Visceral Surgery, 148(5), pp. e3-e8.

[12] Yeung, B. P. M., and Gourlay, T., 2012. "A technical review of flexible endoscopic multitasking platforms". International Journal of Surgery, 10(7), pp. 345-354.

[13] MMI S.P.A., 2020. MMI - Medical Micro Instruments. http://www.mmimicro.com/. Accessed: 04-05-2020.

[14] Auris Health inc., 2020. The Monarch platform. https://www.aurishealth.com/. Accessed: 04-05-2020.

[15] Groenhuis, V., Siepel, F. J., Veltman, J., van Zandwijk, J. K., and Stramigioli, S., 2018. "Stormram 4: An MR Safe Robotic System for Breast Biopsy”. Annals of Biomedical Engineering, 46(10), oct, pp. 1686-1696.

[16] Taylor, R. H., Menciassi, A., Fichtinger, G., Fiorini, P., and Dario, P., 2016. "Medical Robotics and ComputerIntegrated Surgery". In Springer Handbook of Robotics. Springer International Publishing, Cham, pp. 1657-1684.

[17] Mckenna, S. J., Charif, H. N., and Frank, T., 2005. “Towards Video Understanding of Laparoscopic Surgery : Instrument Tracking". In Proc. of Image and Vision Computing, no. November, pp. 2-6.

[18] Intuitive Surgical, 2020. DaVinci Surgical System. www.intuitivesurgical.com. Accessed: 04-05-2020.

[19] Hannaford, B., Rosen, J., Friedman, D. W., King, H., Roan, P., Lei Cheng, Glozman, D., Ji Ma, Kosari, S. N., and White, L., 2013. "Raven-II: An Open Platform for Surgical Robotics Research". IEEE Transactions on Biomedical Engineering, 60(4), apr, pp. 954-959.

[20] Voros, S., Long, J. A., and Cinquin, P., 2007. “Automatic detection of instruments in laparoscopic images: A first step towards high-level command of robotic endoscopic holders". International Journal of Robotics Research, 26(11-12), pp. 1173-1190.

[21] Wolf, R., Duchateau, J., Cinquin, P., and Voros, S., 2011. "3D Tracking of Laparoscopic Instruments Using Statistical and Geometric Modeling”. Medical Image Computing and Computer-Assisted Intervention, 6891, pp. $203-210$.

[22] Garcia-Peraza-Herrera, L. C., Li, W., Fidon, L., Gruijthuijsen, C., Devreker, A., Attilakos, G., Deprest, J., Poorten, E. V., Stoyanov, D., Vercauteren, T., and Ourselin, S., 2017. "ToolNet: Holistically-nested real-time segmentation of robotic surgical tools". In 2017 IEEE/RSJ International Conference on Intelligent Robots and Systems (IROS), IEEE, pp. 5717-5722.

[23] Allan, M., Ourselin, S., Thompson, S., Hawkes, D. J., Kelly, J., and Stoyanov, D., 2013. "Toward detection and localization of instruments in minimally invasive surgery". IEEE Transactions on Biomedical Engineering, 60(4), pp. 1050-1058. 
[24] Reiter, A., Allen, P. K., and Zhao, T., 2012. "Feature Classification for Tracking Articulated Surgical Tools". Medical Image Computing and Computer-Assisted Intervention - MICCAI 2012, pp. 592-600.

[25] Colleoni, E., Moccia, S., Du, X., De Momi, E., and Stoyanov, D., 2019. "Deep Learning Based Robotic Tool Detection and Articulation Estimation with Spatio-Temporal Layers". IEEE Robotics and Automation Letters, 4(3), pp. 27142721.

[26] Bouget, D., Benenson, R., Omran, M., Riffaud, L., Schiele, B., and Jannin, P., 2015. "Detecting Surgical Tools by Modelling Local Appearance and Global Shape". IEEE Transactions on Medical Imaging, 34(12), pp. 2603-2617.

[27] Atkins, M. S., Tien, G., Khan, R. S., Meneghetti, A., and Zheng, B., 2013. "What do surgeons see: Capturing and synchronizing eye gaze for surgery applications". Surgical Innovation, 20(3), pp. 241-248.

[28] García-Mato, D., Lasso, A., Szulewski, A., Pascau, J., and Fichtinger, G., 2017. "3D Gaze Tracking based on Eye and Head Pose Tracking". 10th Hamlyn Symposium on Medical Robotics, pp. 87-88.

[29] , 2020. "Head-Mounted Display Use in Surgery: A Systematic Review". Surgical Innovation, 27(1), feb, pp. 88-100.

[30] Tong, I., Mohareri, O., Tatasurya, S., Hennessey, C., and Salcudean, S., 2015. "A retrofit eye gaze tracker for the da Vinci and its integration in task execution using the da Vinci Research Kit". IEEE International Conference on Intelligent Robots and Systems, 2015-Decem, pp. 2043-2050.

[31] Yip, H. M., Navarro-Alarcon, D., and Liu, Y. H., 2016. "Development of an eye-gaze controlled interface for surgical manipulators using eye-tracking glasses". 2016 IEEE International Conference on Robotics and Biomimetics, ROBIO 2016, pp. 1900-1905.

[32] Konstantinova, J., Jiang, A., Althoefer, K., Dasgupta, P., and Nanayakkara, T., 2014. "Implementation of Tactile Sensing for Palpation in Robot-Assisted Minimally Invasive Surgery: A Review”. IEEE Sensors Journal, 14(8), aug, pp. 2490-2501.

[33] Black, D. G., Hosseinabadi, A. H. H., and Salcudean, S. E., 2020. "6-DOF Force Sensing for the Master Tool Manipulator of the da Vinci Surgical System". IEEE Robotics and Automation Letters, 5(2), apr, pp. 2264-2271.

[34] Piqué, F., Boushaki, M. N., Brancadoro, M., De Momi, E., and Menciassi, A., 2019. "Dynamic Modeling of the da Vinci Research Kit Arm for the Estimation of Interaction Wrench”. 2019 International Symposium on Medical Robotics, ISMR 2019.

[35] Marban, A., Srinivasan, V., Samek, W., Fernández, J., and Casals, A., 2019. "A recurrent convolutional neural network approach for sensorless force estimation in robotic surgery". Biomedical Signal Processing and Control, 50, pp. 134-150.

[36] Wang, Y., Gondokaryono, R., Munawar, A., and Fischer, G. S., 2019. "A Convex Optimization-Based Dynamic Model Identification Package for the da Vinci Research Kit”. IEEE Robotics and Automation Letters, 4(4), pp. 3657-3664.

[37] Sang, H., Yun, J., Monfaredi, R., Wilson, E., Fooladi, H., and Cleary, K., 2017. "External force estimation and implementation in robotically assisted minimally invasive surgery". International Journal of Medical Robotics and Computer Assisted Surgery, 13(2), pp. 1-15.

[38] Krupa, A., Gangloff, J., Doignon, C., De Mathelin, M. F., Morel, G., Leroy, J., Soler, L., and Marescaux, J., 2003. "Autonomous 3-D Positioning of Surgical Instruments in Robotized Laparoscopic Surgery Using Visual Servoing". IEEE Transactions on Robotics and Automation, 19(5), pp. 842-853.

[39] Suárez, C., Acha, B., Serrano, C., Parra, C., and Gómez, T., 2009. "VirSSPA- A virtual reality tool for surgical planning workflow". International Journal of Computer Assisted Radiology and Surgery, 4(2), pp. 133-139.

[40] Das, N., and Yip, M. C., 2020. "Forward Kinematics Kernel for Improved Proxy Collision Checking". IEEE Robotics and Automation Letters, 5(2), apr, pp. 2349-2356.

[41] Sys, G., Eykens, H., Lenaerts, G., Shumelinsky, F., Robbrecht, C., and Poffyn, B., 2017. "Accuracy assessment of surgical planning and three-dimensional-printed patient-specific guides for orthopaedic osteotomies". Proceedings of the Institution of Mechanical Engineers, Part H: Journal of Engineering in Medicine, 231(6), pp. 499-508.

[42] Lee, S. L., Lerotic, M., Vitiello, V., Giannarou, S., Kwok, K. W., Visentini-Scarzanella, M., and Yang, G. Z., 2010. "From medical images to minimally invasive intervention: Computer assistance for robotic surgery". Computerized Medical Imaging and Graphics, 34(1), pp. 33-45.

[43] Roberts, D. W., Strohbehn, J. W., Hatch, J. F., Murray, W., and Kettenberger, H., 1986. "A frameless stereotaxic integration of computerized tomographic imaging and the operating microscope". Journal of Neurosurgery, 65(4), pp. 545-549.

[44] Mochizuki, Y., Hosaka, A., Kamiuchi, H., Nie, J. X., Masamune, K., Hoshina, K., Miyata, T., and Watanabe, T., 2016. "New simple image overlay system using a tablet PC for pinpoint identification of the appropriate site for anastomosis in peripheral arterial reconstruction”. Surgery Today, 46(12), pp. 1387-1393.

[45] Kong, S.-H., Haouchine, N., Soares, R., Klymchenko, A., Andreiuk, B., Marques, B., Shabat, G., Piechaud, T., Diana, M., Cotin, S., and Marescaux, J., 2017. "Robust augmented reality registration method for localization of solid organs' tumors using CT-derived virtual biomechanical model and fluorescent fiducials". Surgical Endoscopy, 31(7), jul, pp. 2863-2871.

[46] Samei, G., Tsang, K., Kesch, C., Lobo, J., Hor, S., Mohareri, O., Chang, S., Goldenberg, S. L., Black, P. C., and 
Salcudean, S., 2020. "A partial augmented reality system with live ultrasound and registered preoperative MRI for guiding robot-assisted radical prostatectomy". Medical Image Analysis, 60, feb, p. 101588.

[47] Katić, D., Wekerle, A. L., Görtler, J., Spengler, P., Bodenstedt, S., Röhl, S., Suwelack, S., Kenngott, H. G., Wagner, M., Müller-Stich, B. P., Dillmann, R., and Speidel, S., 2013. "Context-aware Augmented Reality in laparoscopic surgery". Computerized Medical Imaging and Graphics, 37(2), pp. 174-182.

[48] Qian, L., Deguet, A., and Kazanzides, P., 2018. "ARssist: augmented reality on a head-mounted display for the first assistant in robotic surgery". Healthcare Technology Letters, 5(5), oct, pp. 194-200.

[49] Sgarbura, O., and Vasilescu, C., 2010. "The decisive role of the patient-side surgeon in robotic surgery". Surgical Endoscopy, 24(12), dec, pp. 3149-3155.

[50] Williams, M. A., McVeigh, J., Handa, A. I., and Lee, R., 2020. "Augmented reality in surgical training: a systematic review". Postgraduate Medical Journal, mar, pp. postgradmedj-2020-137600.

[51] Osso Virtual Reality, 2020. The OSSO virtual training system. https://ossovr.com/. Accessed: 04-05-2020.

[52] Wang, S., Parsons, M., Stone-McLean, J., Rogers, P., Boyd, S., Hoover, K., Meruvia-Pastor, O., Gong, M., and Smith, A., 2017. "Augmented Reality as a Telemedicine Platform for Remote Procedural Training". Sensors, 17(10), oct, p. 2294.

[53] Bowthorpe, M., Tavakoli, M., Becher, H., and Howe, R., 2013. "Smith predictor based control in teleoperated imageguided beating-heart surgery". In 2013 IEEE International Conference on Robotics and Automation, pp. 5825-5830.

[54] Wood, N. A., Schwartzman, D., Passineau, M. J., Moraca, R. J., Zenati, M. A., and Riviere, C. N., 2018. "Beatingheart registration for organ-mounted robots". International Journal of Medical Robotics and Computer Assisted Surgery, 14(4), pp. 1-9.

[55] Ruszkowski, A., Mohareri, O., Lichtenstein, S., Cook, R., and Salcudean, S., 2015. "On the feasibility of heart motion compensation on the daVinci $(\mathrm{B}$ surgical robot for coronary artery bypass surgery: Implementation and user studies". Proceedings - IEEE International Conference on Robotics and Automation, 2015-June(June), pp. 4432-4439.

[56] Bowyer, S. A., Davies, B. L., and Rodriguez y Baena, F., 2014. "Active Constraints/Virtual Fixtures: A Survey". IEEE Transactions on Robotics, 30(1), feb, pp. 138-157.

[57] Al Nooryani, A., and Aboushokka, W., 2018. "Rotate-on-Retract Procedural Automation for Robotic-Assisted Percutaneous Coronary Intervention: First Clinical Experience". Case Reports in Cardiology, 2018, dec, pp. 1-3.

[58] Naghibi, H., Hoitzing, W. B., Stramigioli, S., and Abayazid, M., 2018. "A Flexible Endoscopic Sensing Module for Force Haptic Feedback Integration”. In 2018 9th Cairo International Biomedical Engineering Conference (CIBEC), IEEE, pp. 158-161.

[59] Hodgson, S., Tavakoli, M., Lelevé, A., and Tu Pham, M., 2014. "High-fidelity sliding mode control of a pneumatic haptic teleoperation system". Advanced Robotics, 28(10), pp. 659-671.

[60] Ogawa, K., Ohnishi, K., and Ibrahim, Y., 2018. "Development of Flexible Haptic Forceps Based on the ElectroHydraulic Transmission System". IEEE Transactions on Industrial Informatics, 14(8), pp. 1-1.

[61] Molinero, M. B., Dagnino, G., Liu, J., Chi, W., Abdelaziz, M. E. M. K., Kwok, T., Riga, C., and Yang, G., 2019. "Haptic Guidance for Robot-Assisted Endovascular Procedures: Implementation and Evaluation on Surgical Simulator". In 2019 IEEE/RSJ International Conference on Intelligent Robots and Systems (IROS), IEEE, pp. 5398-5403.

[62] Moccia, R., Selvaggio, M., Villani, L., Siciliano, B., and Ficuciello, F., 2019. "Vision-based Virtual Fixtures Generation for Robotic-Assisted Polyp Dissection Procedures". In 2019 IEEE/RSJ International Conference on Intelligent Robots and Systems (IROS), IEEE, pp. 7934-7939.

[63] Van Der Meijden, O. A. J., and Schijven, M. P., 2009. "The value of haptic feedback in conventional and robotassisted minimal invasive surgery and virtual reality training: a current review". Surgical Endoscopy, 23(6), jun, pp. 1180-1190.

[64] Spinelli, A., David, G., Gidaro, S., Carvello, M., Sacchi, M., Montorsi, M., and Montroni, I., 2018. "First experience in colorectal surgery with a new robotic platform with haptic feedback". Colorectal Disease, 20(3), mar, pp. 228-235.

[65] Slawinski, P. R., Taddese, A. Z., Musto, K. B., Obstein, K. L., and Valdastri, P., 2017. "Autonomous Retroflexion of a Magnetic Flexible Endoscope". IEEE Robotics and Automation Letters, 2(3), jul, pp. 1352-1359.

[66] Haro, B. B., Zappella, L., and Vidal, R., 2012. "Surgical gesture classification from video data.". Medical image computing and computer-assisted intervention : MICCAI ... International Conference on Medical Image Computing and Computer-Assisted Intervention, 15(Pt 1), pp. 34-41.

[67] Van Amsterdam, B., Nakawala, H., Momi, E. D., and Stoyanov, D., 2019. "Weakly Supervised Recognition of Surgical Gestures". In 2019 International Conference on Robotics and Automation (ICRA), Vol. 2019-May, IEEE, pp. 9565-9571.

[68] Loukas, C., and Georgiou, E., 2013. "Surgical workflow analysis with Gaussian mixture multivariate autoregressive (GMMAR) models: A simulation study". Computer Aided Surgery, 18(3-4), pp. 47-62.

[69] Beyl, T., Nicolai, P., Comparetti, M. D., Raczkowsky, J., De Momi, E., and Wörn, H., 2016. "Time-of-flight-assisted Kinect camera-based people detection for intuitive human robot cooperation in the surgical operating room". International Journal of Computer Assisted Radiology and Surgery, 11(7), pp. 1329-1345. 
[70] Ahmidi, N., Tao, L., Sefati, S., Gao, Y., Lea, C., Haro, B. B., Zappella, L., Khudanpur, S., Vidal, R., and Hager, G. D., 2017. "A Dataset and Benchmarks for Segmentation and Recognition of Gestures in Robotic Surgery". IEEE Transactions on Biomedical Engineering, 64(9), pp. 2025-2041.

[71] DiPietro, R., Ahmidi, N., Malpani, A., Waldram, M., Lee, G. I., Lee, M. R., Vedula, S. S., and Hager, G. D., 2019. "Segmenting and classifying activities in robot-assisted surgery with recurrent neural networks". International Journal of Computer Assisted Radiology and Surgery, 14(11), pp. 2005-2020.

[72] Nageotte, F., Zanne, P., Doignon, C., and De Mathelin, M., 2009. "Stitching planning in laparoscopic surgery: Towards robot-assisted suturing". International Journal of Robotics Research, 28(10), pp. 1303-1321.

[73] Jackson, R. C., Desai, V., Castillo, J. P., and Çavuşoğlu, M. C., 2016. "Needle-tissue interaction force state estimation for robotic surgical suturing". IEEE International Conference on Intelligent Robots and Systems, 2016-November, pp. 3659-3664.

[74] Zhong, F., Wang, Y., Wang, Z., and Liu, Y. H., 2019. "Dual-Arm Robotic Needle Insertion with Active Tissue Deformation for Autonomous Suturing". IEEE Robotics and Automation Letters, 4(3), pp. 2669-2676.

[75] Pedram, S. A., Ferguson, P., Ma, J., Dutson, E., and Rosen, J., 2017. "Autonomous suturing via surgical robot: An algorithm for optimal selection of needle diameter, shape, and path". Proceedings - IEEE International Conference on Robotics and Automation, pp. 2391-2398.

[76] Staub, C., Osa, T., Knoll, A., and Bauernschmitt, R., 2010. "Automation of tissue piercing using circular needles and vision guidance for computer aided laparoscopic surgery". Proceedings - IEEE International Conference on Robotics and Automation, pp. 4585-4590.

[77] Watanabe, K., Kanno, T., Ito, K., and Kawashima, K., 2018. "Single-Master Dual-Slave Surgical Robot with Automated Relay of Suture Needle". IEEE Transactions on Industrial Electronics, 65(8), pp. 6343-6351.

[78] Schulman, J., and Tayson-frederick, M., 2013. "A Case Study of Trajectory Transfer Through Non-Rigid Registration for a Simplified Suturing Scenario”. In 2013 IEEE/RSJ International Conference on Intelligent Robots and Systems, pp. 4111-4117.

[79] Sen, S., Garg, A., Gealy, D. V., McKinley, S., Jen, Y., and Goldberg, K., 2016. “Automating multi-throw multilateral surgical suturing with a mechanical needle guide and sequential convex optimization". In 2016 IEEE International Conference on Robotics and Automation (ICRA), IEEE, pp. 4178-4185.

[80] Chow, D. L., and Newman, W., 2013. "Improved knot-tying methods for autonomous robot surgery". IEEE International Conference on Automation Science and Engineering, pp. 461-465.

[81] Mayer, H., Gomez, F., Wierstra, D., Nagy, I., Knoll, A., and Schmidhuber, J., 2008. "A system for robotic heart surgery that learns to tie knots using recurrent neural networks". Advanced Robotics, 22(13-14), pp. 1521-1537.

[82] Knoll, A., Mayer, H., Staub, C., and Bauernschmitt, R., 2012. "Selective automation and skill transfer in medical robotics: a demonstration on surgical knot-tying". The International Journal of Medical Robotics and Computer Assisted Surgery, 8(4), dec, pp. 384-397.

[83] Chow, D. L., and Newman, W., 2015. "Trajectory optimization of robotic suturing". IEEE Conference on Technologies for Practical Robot Applications, TePRA, 2015-Augus, pp. 1-6.

[84] Leonard, S., Wu, K. L., Kim, Y., Krieger, A., and Kim, P. C., 2014. "Smart tissue anastomosis robot (STAR): A vision-guided robotics system for laparoscopic suturing". IEEE Transactions on Biomedical Engineering, 61(4), pp. 1305-1317.

[85] Krieger, A., Opfermann, J., and Kim, P. C. W., 2017. "Development and Feasibility of a Robotic Laparoscopic Clipping Tool for Wound Closure and Anastomosis". Journal of Medical Devices, 12(1), p. 011005.

[86] Jansen, R., Hauser, K., Chentanez, N., Van Der Stappen, F., and Goldberg, K., 2009. "Surgical retraction of nonuniform deformable layers of tissue: 2D robot grasping and path planning". 2009 IEEE/RSJ International Conference on Intelligent Robots and Systems, IROS 2009, pp. 4092-4097.

[87] Patil, S., and Alterovitz, R., 2010. "Toward automated tissue retraction in robot-assisted surgery". Proceedings - IEEE International Conference on Robotics and Automation, pp. 2088-2094.

[88] Elek, R., Nagy, T. D., Nagy, D., Garamvölgyi, T., Takács, B., Galambos, P., Tar, J. K., Rudas, I. J., and Haidegger, T., 2017. "Towards surgical subtask automation-blunt dissection". In INES 2017 - IEEE 21st International Conference on Intelligent Engineering Systems, Proceedings, Vol. 2017-Janua, pp. 253-257.

[89] Nagy, T. D., Takacs, M., Rudas, I. J., and Haidegger, T., 2018. "Surgical subtask automation — Soft tissue retraction". In 2018 IEEE 16th World Symposium on Applied Machine Intelligence and Informatics (SAMI), IEEE, pp. 55-60.

[90] Trejos, A. L., Jayender, J., Perri, M. T., Naish, M. D., Patel, R. V., and Malthaner, R. A., 2009. "Robot-assisted tactile sensing for minimally invasive tumor localization". International Journal of Robotics Research, 28(9), pp. 1118-1133.

[91] Back, J., Dasgupta, P., Seneviratne, L., Althoefer, K., and Liu, H., 2015. "Feasibility study- novel optical soft tactile array sensing for minimally invasive surgery". IEEE International Conference on Intelligent Robots and Systems, 2015-Decem(c), pp. 1528-1533.

[92] McKinley, S., Garg, A., Sen, S., Kapadia, R., Murali, A., Nichols, K., Lim, S., Patil, S., Abbeel, P., Okamura, A. M., and Goldberg, K., 2015. "A single-use haptic palpation probe for locating subcutaneous blood vessels in 
robot-assisted minimally invasive surgery”. IEEE International Conference on Automation Science and Engineering, pp. 1151-1158.

[93] Campisano, F., Ozel, S., Ramakrishnan, A., Dwivedi, A., Gkotsis, N., Onal, C. D., and Valdastri, P., 2017. "Towards a soft robotic skin for autonomous tissue palpation". Proceedings - IEEE International Conference on Robotics and Automation, pp. 6150-6155.

[94] Bajo, A., and Simaan, N., 2016. "Hybrid motion/force control of multi-backbone continuum robots". The International Journal of Robotics Research, 35(4), apr, pp. 422-434.

[95] Ayvali, E., Ansari, A., Wang, L., Simaan, N., and Choset, H., 2017. "Utility-Guided Palpation for Locating Tissue Abnormalities". IEEE Robotics and Automation Letters, 2(2), apr, pp. 864-871.

[96] Nichols, K. A., and Okamura, A. M., 2015. "Methods to Segment Hard Inclusions in Soft Tissue During Autonomous Robotic Palpation". IEEE Transactions on Robotics, 31(2), apr, pp. 344-354.

[97] Ayvali, E., Srivatsan, R. A., Wang, L., Roy, R., Simaan, N., and Choset, H., 2016. "Using Bayesian optimization to guide probing of a flexible environment for simultaneous registration and stiffness mapping". In 2016 IEEE International Conference on Robotics and Automation (ICRA), IEEE, pp. 931-936.

[98] Chalasani, P., Wang, L., Roy, R., Simaan, N., Taylor, R. H., and Kobilarov, M., 2016. "Concurrent nonparametric estimation of organ geometry and tissue stiffness using continuous adaptive palpation". In 2016 IEEE International Conference on Robotics and Automation (ICRA), IEEE, pp. 4164-4171.

[99] Constanciel, E., N'Djin, W. A., Bessiere, F., Chavrier, F., Grinberg, D., Vignot, A., Chevalier, P., Chapelon, J. Y., and Lafon, C., 2013. "Design and evaluation of a transesophageal HIFU probe for ultrasound-guided cardiac ablation: simulation of a HIFU mini-maze procedure and preliminary ex vivo trials". IEEE Transactions on Ultrasonics, Ferroelectrics, and Frequency Control, 60(9), sep, pp. 1868-1883.

[100] Wang, H., Kang, W., Carrigan, T., Bishop, A., Rosenthal, N., Arruda, M., and Rollins, A. M., 2011. "In vivo intracardiac optical coherence tomography imaging through percutaneous access: toward image-guided radio-frequency ablation". Journal of Biomedical Optics, 16(11), p. 110505.

[101] Yang, L., Wen, R., Qin, J., Chui, C. K., Lim, K. B., and Chang, S. K. Y., 2010. "A robotic system for overlapping radiofrequency ablation in large tumor treatment". IEEE/ASME Transactions on Mechatronics, 15(6), pp. 887-897.

[102] Su, B., Tang, J., and Liao, H., 2015. "Automatic laser ablation control algorithm for an novel endoscopic laser ablation end effector for precision neurosurgery". IEEE International Conference on Intelligent Robots and Systems, 2015-December, pp. 4362-4367.

[103] Sarli, N., Del Giudice, G., De, S., Dietrich, M. S., Herrell, S. D., and Simaan, N., 2018. "Preliminary Porcine In Vivo Evaluation of a Telerobotic System for Transurethral Bladder Tumor Resection and Surveillance ". Journal of Endourology, 32(6), pp. 516-522.

[104] Alambeigi, F., Wang, Z., hui Liu, Y., Taylor, R. H., and Armand, M., 2018. "Toward Semi-autonomous Cryoablation of Kidney Tumors via Model-Independent Deformable Tissue Manipulation Technique". Annals of Biomedical Engineering, 46(10), pp. 1650-1662.

[105] Taktak, S., Jones, P., Haq, A., Rai, B. P., and Somani, B. K., 2018. "Aquablation: a novel and minimally invasive surgery for benign prostate enlargement". Therapeutic Advances in Urology, 10(6), jun, pp. 183-188.

[106] Martin, J. W., Slawinski, P. R., Scaglioni, B., Norton, J. C., Valdastri, P., and Obstein, K. L., 2019. "Assistive autonomoy in colonoscopy: Propulsion of a magnetic flexible endoscope". Gastrointestinal Endoscopy, 89(6), pp. AB76-AB77.

[107] Okamura, A. M., Simone, C., and O'Leary, M. D., 2004. "Force modeling for needle insertion into soft tissue". IEEE Transactions on Biomedical Engineering, 51(10), pp. 1707-1716.

[108] Osa, T., Abawi, C. F., Sugita, N., Chikuda, H., Sugita, S., Ito, H., Moro, T., Takatori, Y., Tanaka, S., and Mitsuishi, M., 2014. "Autonomous penetration detection for bone cutting tool using demonstration-based learning". Proceedings IEEE International Conference on Robotics and Automation, pp. 290-296.

[109] Yip, M. C., Lowe, D. G., Salcudean, S. E., Rohling, R. N., and Nguan, C. Y., 2012. "Tissue tracking and registration for image-guided surgery". IEEE Transactions on Medical Imaging, 31(11), pp. 2169-2182.

[110] Peterlík, I., Courtecuisse, H., Rohling, R., Abolmaesumi, P., Nguan, C., Cotin, S., and Salcudean, S., 2018. "Fast elastic registration of soft tissues under large deformations". Medical Image Analysis, 45, pp. 24-40.

[111] Navarro-Alarcon, D., Yip, H. M., Wang, Z., Liu, Y. H., Zhong, F., Zhang, T., and Li, P., 2016. “Automatic 3D Manipulation of Soft Objects by Robotic Arms with an Adaptive Deformation Model". IEEE Transactions on Robotics, 32(2), pp. 429-441.

[112] Alambeigi, F., Wang, Z., Hegeman, R., Liu, Y. H., and Armand, M., 2019. "Autonomous data-driven manipulation of unknown anisotropic deformable tissues using unmodelled continuum manipulators". IEEE Robotics and Automation Letters, 4(2), pp. 254-261.

[113] Pappone, C., Ciconte, G., Vicedomini, G., Mangual, J. O., Li, W., Conti, M., Giannelli, L., Lipartiti, F., McSpadden, L., Ryu, K., Guazzi, M., Menicanti, L., and Santinelli, V., 2018. "Clinical Outcome of Electrophysiologically Guided Ablation for Nonparoxysmal Atrial Fibrillation Using a Novel Real-Time 3-Dimensional Mapping Technique". Cir- 
culation: Arrhythmia and Electrophysiology, 11(3), mar.

[114] Decker, R., Shademan, A., Opfermann, J., Leonard, S., Kim, P. C. W., and Krieger, A., 2015. "Performance evaluation and clinical applications of 3D plenoptic cameras". In Next-Generation Robotics II; and Machine Intelligence and Bio-inspired Computation: Theory and Applications IX, no. June, p. 94940B.

[115] Shademan, A., Decker, R. S., Opfermann, J., Leonard, S., Kim, P. C., and Krieger, A., 2016. "Plenoptic cameras in surgical robotics: Calibration, registration, and evaluation”. Proceedings - IEEE International Conference on Robotics and Automation, 2016-June, pp. 708-714.

[116] Bloch, E., Thurin, B., Keane, P., Nousias, S., Bergeles, C., and Ourselin, S., 2018. "Retinal fundus imaging with a plenoptic sensor". In Ophthalmic Technologies XXVIII, Vol. 1047429, SPIE, p. 81.

[117] Clancy, N. T., Jones, G., Maier-Hein, L., Elson, D. S., and Stoyanov, D., 2020. "Surgical spectral imaging”. Medical Image Analysis, 63, jul, p. 101699.

[118] Yu, L., Hao, L., Meiqiong, T., Jiaoqi, H., Wei, L., Jinying, D., Xueping, C., Weiling, F., and Yang, Z., 2019. “The medical application of terahertz technology in non-invasive detection of cells and tissues: opportunities and challenges". RSC Advances, 9(17), pp. 9354-9363.

[119] Speidel, S., Kroehnert, A., Bodenstedt, S., Kenngott, H., Müller-Stich, B., and Dillmann, R., 2015. "Image-based tracking of the suturing needle during laparoscopic interventions". In Medical Imaging 2015: Image-Guided Procedures, Robotic Interventions, and Modeling, R. J. Webster and Z. R. Yaniv, eds., Vol. 9415, p. 94150B.

[120] Gu, Y., Hu, Y., Zhang, L., Yang, J., and Yang, G.-Z., 2018. “Cross-Scene Suture Thread Parsing for Robot Assisted Anastomosis based on Joint Feature Learning”. In 2018 IEEE/RSJ International Conference on Intelligent Robots and Systems (IROS), pp. 769-776.

[121] Jackson, R. C., Yuan, R., Chow, D.-L., Newman, W. S., and Cavusoglu, M. C., 2018. "Real-Time Visual Tracking of Dynamic Surgical Suture Threads”. IEEE Transactions on Automation Science and Engineering, 15(3), jul, pp. 10781090.

[122] Padoy, N., and Hager, G. D., 2011. "3D thread tracking for robotic assistance in tele-surgery”. IEEE International Conference on Intelligent Robots and Systems, pp. 2102-2107.

[123] D’Ettorre, C., Dwyer, G., Du, X., Chadebecq, F., Vasconcelos, F., De Momi, E., and Stoyanov, D., 2018. “Automated Pick-Up of Suturing Needles for Robotic Surgical Assistance”. In 2018 IEEE International Conference on Robotics and Automation (ICRA), Vol. 1, pp. 1370-1377.

[124] Beigi, P., Rohling, R., Salcudean, T., Lessoway, V. A., and Ng, G. C., 2017. "Detection of an invisible needle in ultrasound using a probabilistic SVM and time-domain features”. Ultrasonics, 78, pp. 18-22.

[125] Mathiassen, K., Dall'Alba, D., Muradore, R., Fiorini, P., and Elle, O. J., 2017. "Robust Real-Time Needle Tracking in 2-D Ultrasound Images Using Statistical Filtering”. IEEE Transactions on Control Systems Technology, 25(3), pp. 966-978.

[126] Zhong, F., and Liu, Y., 2018. "Image-based 3D pose reconstruction of surgical needle for robot-assisted laparoscopic suturing". Chinese Journal of Electronics, 27(3), pp. 476-482.

[127] Abayazid, M., Roesthuis, R. J., Reilink, R., and Misra, S., 2013. "Integrating deflection models and image feedback for real-time flexible needle steering”. IEEE Transactions on Robotics, 29(2), pp. 542-553.

[128] Vrooijink, G. J., Abayazid, M., Patil, S., Alterovitz, R., and Misra, S., 2014. "Needle path planning and steering in a three-dimensional non-static environment using two-dimensional ultrasound images". International Journal of Robotics Research, 33(10), pp. 1361-1374.

[129] Patel, N. A., van Katwijk, T., Gang Li, Moreira, P., Weijian Shang, Misra, S., and Fischer, G. S., 2015. “Closed-loop asymmetric-tip needle steering under continuous intraoperative MRI guidance”. In 2015 37th Annual International Conference of the IEEE Engineering in Medicine and Biology Society (EMBC), IEEE, pp. 4869-4874.

[130] Moreira, P., Boskma, K. J., and Misra, S., 2017. "Towards MRI-guided flexible needle steering using fiber Bragg grating-based tip tracking". In 2017 IEEE International Conference on Robotics and Automation (ICRA), IEEE, pp. 4849-4854.

[131] Shahriari, N., Georgiadis, J. R., Oudkerk, M., and Misra, S., 2018. "Hybrid control algorithm for flexible needle steering: Demonstration in phantom and human cadaver". PLOS ONE, 13(12), dec, p. e0210052.

[132] Fu, M., Kuntz, A., Webster, R. J., and Alterovitz, R., 2018. "Safe Motion Planning for Steerable Needles Using Cost Maps Automatically Extracted from Pulmonary Images”. In 2018 IEEE/RSJ International Conference on Intelligent Robots and Systems (IROS), IEEE, pp. 4942-4949.

[133] Fagogenis, G., Mencattelli, M., Machaidze, Z., Rosa, B., Price, K., Wu, F., Weixler, V., Saeed, M., Mayer, J. E., and Dupont, P. E., 2019. "Autonomous robotic intracardiac catheter navigation using haptic vision". Science Robotics, 4(29), apr, p. eaaw 1977.

[134] Saeidi, H., Le, H. N. D., Opfermann, J. D., Leonard, S., Kim, A., Hsieh, M. H., Kang, J. U., and Krieger, A., 2019. "Autonomous Laparoscopic Robotic Suturing with a Novel Actuated Suturing Tool and 3D Endoscope". In 2019 International Conference on Robotics and Automation (ICRA), Vol. 2019-May, IEEE, pp. 1541-1547.

[135] Havaei, M., Davy, A., Warde-Farley, D., Biard, A., Courville, A., Bengio, Y., Pal, C., Jodoin, P. M., and Larochelle, 
H., 2017. "Brain tumor segmentation with Deep Neural Networks". Medical Image Analysis, 35, pp. 18-31.

[136] Hu, P., Wu, F., Peng, J., Liang, P., and Kong, D., 2016. “Automatic 3D liver segmentation based on deep learning and globally optimized surface evolution”. Physics in Medicine and Biology, 61(24), dec, pp. 8676-8698.

[137] Qiu, W., Yuan, J., Ukwatta, E., Sun, Y., Rajchl, M., and Fenster, A., 2014. "Prostate segmentation: an efficient convex optimization approach with axial symmetry using 3-D TRUS and MR images.". IEEE transactions on medical imaging, 33(4), apr, pp. 947-60.

[138] Li, X., Chen, H., Qi, X., Dou, Q., Fu, C.-W., and Heng, P.-A., 2018. "H-DenseUNet: Hybrid Densely Connected UNet for Liver and Tumor Segmentation From CT Volumes". IEEE Transactions on Medical Imaging, 37(12), dec, pp. 2663-2674.

[139] BrainLab, 2020. iPlan RT. https://www.brainlab.com/radiosurgery-products/iplan-rt-treatment-planning-software/. Accessed: 04-05-2020.

[140] Opfermann, J. D., Leonard, S., Decker, R. S., Uebele, N. A., Bayne, C. E., Joshi, A. S., and Krieger, A., 2017. "Semi-autonomous electrosurgery for tumor resection using a multi-degree of freedom electrosurgical tool and visual servoing”. In 2017 IEEE/RSJ International Conference on Intelligent Robots and Systems (IROS), pp. 3653-3660.

[141] Nichols, K. A., and Okamura, A. M., 2013. "Autonomous robotic palpation: Machine learning techniques to identify hard inclusions in soft tissues". Proceedings - IEEE International Conference on Robotics and Automation, pp. 43844389.

[142] McKinley, S., Garg, A., Sen, S., Gealy, D. V., McKinley, J. P., Jen, Y., Guo, M., Boyd, D., and Goldberg, K., 2016. “An interchangeable surgical instrument system with application to supervised automation of multilateral tumor resection". IEEE International Conference on Automation Science and Engineering, 2016-Novem, pp. 821-826.

[143] USA, Department of Defense, 2017. Toward a next-generation trauma care capability: Foundational research for autonomous, unmanned, and robotics development of medical technologies forward award.

[144] O’Sullivan, S., Nevejans, N., Allen, C., Blyth, A., Leonard, S., Pagallo, U., Holzinger, K., Holzinger, A., Sajid, M. I., and Ashrafian, H., 2019. "Legal, regulatory, and ethical frameworks for development of standards in artificial intelligence (AI) and autonomous robotic surgery". The International Journal of Medical Robotics and Computer Assisted Surgery, 15(1), p. e1968.

[145] European Parliament, 2017. “"'European Parliament resolution of 16 February 2017 number:P8 TA 20170051 '”'.

[146] European Parliament, 2018. "Comprehensive European industrial policy on artificial intelligence and robotics".

[147] O’Sullivan, S., Leonard, S., Holzinger, A., Allen, C., Battaglia, F., Nevejans, N., Leeuwen, F. W., Sajid, M. I., Friebe, M., Ashrafian, H., Heinsen, H., Wichmann, D., and Hartnett, M., 2019. "Anatomy 101 for AI-driven robotics: Explanatory, ethical and legal frameworks for development of cadaveric skills training standards in autonomous robotic surgery/autopsy". The International Journal of Medical Robotics and Computer Assisted Surgery, may, p. e2020.

[148] Jamjoom, A. A. B., Jamjoom, A. M. A., and Marcus, H. J., 2020. "Exploring public opinion about liability and responsibility in surgical robotics". Nature Machine Intelligence, 2(4), apr, pp. 194-196.

[149] Shah, R., and Nagaraja, S., 2019. "Privacy with surgical robotics: challenges in applying contextual privacy theory".

[150] Haidegger, T., 2019. "Autonomy for surgical robots: Concepts and paradigms". IEEE Transactions on Medical Robotics and Bionics, 1(2), pp. 65-76.

[151] Datteri, E., 2013. "Predicting the long-term effects of human-robot interaction: A reflection on responsibility in medical robotics". Science and engineering ethics, 19(1), pp. 139-160.

[152] Stahl, B. C., and Coeckelbergh, M., 2016. "Ethics of healthcare robotics: Towards responsible research and innovation”. Robotics and Autonomous Systems, 86, pp. 152-161. 